\title{
Specular Null Polarization Theory: Applications to Radar Meteorology
}

\author{
John C. Hubbert and V. N. Bringi
}

\begin{abstract}
Specular null polarization theory (SNPT) has been recently introduced for the case of coherent scattering where a $2 \times 2$ scattering matrix is sufficient to describe the scattering process. In this paper, SNPT is extended to the case of incoherent scattering. Optimum polarization states are derived and the results are discussed in relation to the classic radar optimum polarizations. In traditional radar polarimetry, modeling of the radar receive/transmit network is included in the radar voltage equation and consequently this affects the optimum polarizations and polarization responses of scatterers. SNPT eliminates this effect and therefore allows for a more direct analysis of scatterers. Modeling of ensembles of precipitation particles is used to illustrate the results of the analysis.
\end{abstract}

\section{INTRODUCTION}

$\mathbf{T}$ HE starting point for the development of radar polarimetry is the radar voltage equation [1]:

$$
\begin{aligned}
\mathcal{V} & =\mathbf{h}_{r}^{T} \mathbf{E}^{r} \\
& =\mathbf{h}_{r}^{T} \mathbf{S} \mathbf{E}^{i} \\
& =\mathbf{h}_{r}^{T} \mathbf{S} \mathbf{h}_{t}
\end{aligned}
$$

where $\mathbf{E}^{r}$, the electric field incident on the radar, $\mathbf{h}_{t}$, the transmit polarization vector of the radar, and $\mathbf{h}_{r}$, the receive polarization vector of the radar, are all described in the same coordinate system (i.e., the definition of the horizontal and vertical unit vectors remains the same for both forward and backward propagation directions) which is shown in Fig. 1 for monostatic radar. This is referred to as the radar alignment convention (RAC) or backscatter alignment convention (BSA) by Ulaby and van Zyl [2]. The superscript $T$ in (1) denotes transpose, $\mathbf{E}^{i}$ is the incident (or transmitted) electric field and $\mathbf{S}$ is the $2 \times 2$ radar (or Sinclair) scattering matrix. Additionally, the radar reception polarization vector is defined as that polarization that the radar transmits so that for monostatic radar $\mathbf{h}_{r}=\mathbf{h}_{\boldsymbol{t}}$. From (1) it is easy to derive the change of basis transformation [1], [3], [4]

$$
\begin{aligned}
\tilde{\mathbf{S}} & =\mathbf{U}^{T} \mathbf{S U} \\
& =\left[\begin{array}{ll}
S_{11} & S_{12} \\
S_{21} & S_{22}
\end{array}\right]
\end{aligned}
$$

where $\mathbf{U}$ is the field transformation matrix and is defined as

$$
\mathbf{U}=\Gamma\left[\begin{array}{cc}
\varrho & -\varrho^{*} \chi^{*} \\
\varrho \chi & \varrho^{*}
\end{array}\right]
$$

Manuscript received January 6, 1995; accepted February 27, 1996. This work was supported by the National Science Foundation under Grants ATM8915141 and ATM-9214864.

The authors are with the Department of Electrical Engineering, Colorado State University, Fort Collins, CO 80523 USA.

Publisher Item Identifier S 0196-2892(96)05044-9. where $*$ signifies complex conjugation and $\Gamma=\left(1+\chi \chi^{*}\right)^{-0.5}$. As shown in [5] the phase term $\varrho=e^{-j \tan ^{-1}(\tan \phi \tan \epsilon)}$ is necessary to maintain a constant phase difference between the elliptic basis polarization vectors and is easily derived from the geometric form for $\mathbf{U}$. The $\phi$ and $\epsilon$ represent the tilt and ellipticity angles, respectively. From (2) optimum polarizations are derived [1], [4]. Optimum polarizations are those transmit polarization states that produce power extrema in either the receive copolar or cross-polar power [4]. Optimum polarizations may also be developed starting with the equation that directly relates the incident and scattered polarization ratios in a local right handed coordinate system convention (LRH) which is shown in Fig. 2. The LRH is typically used in optic polarimetry [6], [7] and is also referred to as the forward scattering alignment convention (FSA) by Ulaby and van Zyl [2]. From Figs. 1 and 2 it is seen that for the backscatter direction $E_{V^{\prime}}^{s}=E_{V}^{s}$ and $E_{H^{\prime}}^{s}=-E_{H}^{s}$. Accordingly, there are two possible polarization ratios for backscatter depending on the coordinate system convention adopted. For the LRH

$$
\chi^{s_{-} \mathrm{LRH}}=\frac{E_{V^{\prime}}^{s}}{E_{H^{\prime}}^{s}}
$$

and for the RAC

$$
\chi^{s \_\mathrm{RAC}}=\frac{E_{V}^{s}}{E_{H}^{s}} .
$$

The two polarization ratios are related by

$$
\chi^{s_{-} \mathrm{LRH}}=-\chi^{s_{-} \mathrm{RAC}} .
$$

The bilinear polarization transfer function that relates incident and scattered polarization ratios in the LRH is (see Appendix A)

$$
\chi^{s_{-} \mathrm{LRH}}=\frac{S_{\mathrm{VH}}+\chi^{i} S_{\mathrm{VV}}}{-S_{\mathrm{HH}}-\chi^{i} S_{\mathrm{HV}}} .
$$

Equation (7) can be used as a common starting point to develop and compare the optimum polarizations of radar, optic and specular null polarization theories (SNPT) as was done by Hubbert [5]. The optimum polarizations in traditional radar polarimetry, henceforth called Kennaugh's polarization theory (KPT), called cross-polar nulls are the equivalent of the eigenpolarizations in optic polarimetry. We note however, that the optic eigenpolarizations are not the same polarization states as the KPT cross-polar nulls. In other words, for the same coherent scatterer different transmit polarizations will produce nulls in the "cross-polar" powers as is defined in optic polarimetry, KPT and SNPT. Using (7) the eigenpolarizations 

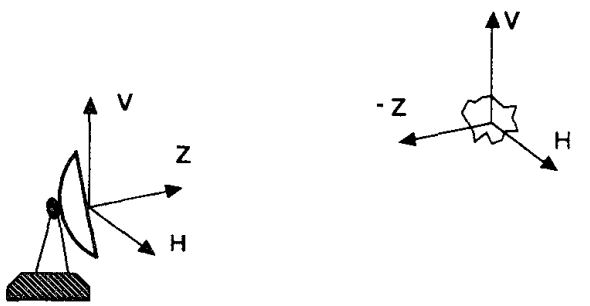

Fig. 1. The radar alignment convention (RAC) for the monostatic case as used in classic radar polarimetry.

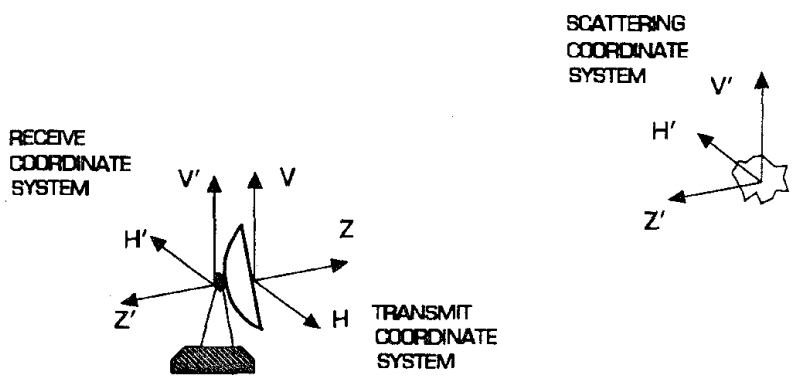

Fig. 2. The LRH used to describe the transmit polarization state, the backscattered wave, and the receive polarization state. $H^{\prime}=-H$, $V^{\prime}=V, Z^{\prime}=-Z$

as defined in optic polarimetry are found by setting $\chi^{\text {s_LRH }}=$ $\chi^{i}=\chi$ and solving for $\chi$ as is done in [7]. This is equivalent to defining $\chi$ as the optic copolar reception polarization state. If one wishes to find the "eigenpolarizations" of KPT, one sets $\chi^{i}=\chi$ and $\chi^{s-\mathrm{LRH}}=-\chi^{*}$ and solves for $\chi$. This is equivalent to defining the $-\chi^{*}$ as the radar copolar reception polarization state. The reader is reminded that the optimum reception state, i.e., that reception polarization state that maximizes copolar power return, for a radar that transmits $\chi$ polarization is $\chi^{s-\mathrm{RAC}}=\chi^{*}$ when using the RAC. If the LRH is employed the polarization ratio becomes $\chi^{s_{-} \mathrm{LRH}}=$ $-\chi^{*}$. Thus we see that the complex conjugation that appears in both $\chi^{s_{-} \mathrm{RAC}}$ and $\chi^{s_{-} \mathrm{LRH}}$ is a result of KPT where the receive network of the radar is modeled together with the scatterer [3], [8], [9].

Obviously, $\chi^{\text {s.LRH }}$ and $\chi^{i}$ can be arbitrarily defined. Hubbert [5] proposed the choice $\chi^{i}=\chi$ and $\chi^{s_{-} \mathrm{LRH}}=-\chi$ which is equivalent to defining $-\chi$ as the copolar reception polarization state. Physically this means that

$$
\frac{E_{V}^{i}}{E_{H}^{i}}=-\frac{E_{V^{\prime}}^{s}}{E_{H^{\prime}}^{s}}
$$

or in the RAC

$$
\frac{E_{V}^{i}}{E_{H}^{i}}=\frac{E_{V}^{s}}{E_{H}^{s}} .
$$

It is now apparent why the SNPT choice of "copolar" polarization state is advantageous. For forward scattering a polarization state is an eigenpolarization if $\chi^{f s}=\chi^{i}$ where the superscript ${ }^{f s}$ denotes forward scattering. It seems natural then to require (9) (i.e., $\chi^{s_{-} \mathrm{RAC}}=\chi^{i}$ ) for backscatter eigenpolarizations using the RAC. Using this definition and solving (7) for $\chi$ yields the "eigenpolarizations" for SNPT. With the copolar reception state defined as $\chi$ in the RAC, it follows that the voltage received in the $\mathrm{H}-\mathrm{V}$ (horizontal and vertical) basis in this so defined "copolar". channel may be expressed as [5]

$$
\nu=\Gamma\left[\begin{array}{l}
1 \\
\chi
\end{array}\right]^{\dagger}\left[\begin{array}{ll}
S_{\mathrm{HH}} & S_{\mathrm{HV}} \\
S_{\mathrm{VH}} & S_{\mathrm{VV}}
\end{array}\right]\left[\begin{array}{l}
1 \\
\chi
\end{array}\right]
$$

or more compactly

$$
\mathcal{V}=\mathrm{g}^{\dagger} \mathrm{Sg}
$$

where ${ }^{\dagger}$ denotes Hermitian adjoint and $\mathrm{g}$ denotes the polarization vector of the radar either as a receiver or a transmitter in the RAC. We emphasize that (11) is not meant to model fixed polarization monostatic radar; however, a polarization agile radar such as POLDIRAD at DLR (the German Aerospace Agency) at Oberphaffenhofen, Germany can be programmed to obey (11). The transmit and receive polarization states of this radar can be set independently to arbitrary elliptic polarization states. Equation (11) is termed the "specular voltage equation." From (10) the change of basis formula for SNPT is found as

$$
\begin{aligned}
\ddot{\tilde{\mathbf{S}}} & =\mathbf{U}^{-1} \ddot{\mathbf{S}} \mathbf{U} \\
& =\left[\begin{array}{ll}
\ddot{S}_{11} & \ddot{S}_{12} \\
\ddot{S}_{21} & \ddot{S}_{22}
\end{array}\right]
\end{aligned}
$$

where $\ddot{\mathbf{S}}$ is the SNPT scattering matrix. The SNPT scattering matrix is identical to the KPT scattering matrix for linear polarizations only since for linear polarizations $\chi$ is real and therefore $\mathrm{U}^{-1}=\mathrm{U}^{\mathrm{T}}$. For completeness we also give the three eigenvalue statements: for optic polarimetry (using the LRH)

$$
\dot{\mathbf{S}} \mathbf{x}=\lambda \mathbf{x}
$$

for KPT (using the RAC)

$$
\mathbf{S x}=\lambda \mathbf{x}^{*}
$$

and for SNPT (using the RAC)

$$
\ddot{\mathbf{S}} \mathbf{x}=\lambda \mathbf{x} .
$$

Note the complex conjugation that appears on the right hand side of the KPT eigenvalue statement (14) which is a direct result of modeling the receive radar network.

The three fields, optic polarimetry, radar polarimetry, and SNPT, may also be compared via the "voltage equations" associated with each. For a comparison of voltage equations, see Appendix B.

Hubbert [5] has developed SNPT for the case of coherent scattering. In this paper SNPT is extented to incoherent scattering through the use of an ensemble averaged covariance matrix. Optimum polarizations are derived and they are compared to the optimum polarizations that are derived using traditional radar polarimetry. For a detailed description of the existing theory for deriving optimum polarizations via the covariance matrix see [10]-[12]. Optimum polarizations are those incident polarization states which minimize or maximize copolar power or cross-polar power. Tragl et al. [11] express the copolar and cross-polar power functions in real Hermitian quadratic forms. This allows the optimum polarizations to be 
calculated via the eigenvalues of a $3 \times 3$ real symmetric matrix for cross-polar extrema or via the Lagrange multiplier method for the copolar extrema. This same approach is followed here for SNPT. First, the nonreciprocal case is solved and then the simplification $S_{\mathrm{HV}}=S_{\mathrm{VH}}$ is introduced. Next, ensembles of precipitation particles are modeled and covariance matrices are constructed using the transition ( $T$-) matrix method [13]. The covariance matrices of typical precipitation particles are used to illustrate the results of the developed theory. Specifically, we demonstrate how modeling of the radar in KPT affects polarization responses and optimum polarizations and show that SNPT eliminates these effects thus allowing for a better characterization of scatterers.

\section{RADAR MOdeling AND POLARIMETriC Signatures}

With the advent of polarimetric agile radars, interest in polarimetric signatures, optimum polarizations and elliptical polarization basis has increased [14]-[17]. When analyzing the chacteristics of scatterers it is important to understand how modeling the radar in KPT effects the various polarization signatures, i.e., it is of interest to identify and separate those polarization features that can be attributed to the scatterer(s) from those caused by the modeling of the radar. With this in mind SNPT was formulated.

The copolar reception polarization state for SNPT is defined so that the return from a specular scatterer, such as a spherical raindrop, will always be completely received by the copolar channel. A specular scatterer is described in the $\mathrm{H}-\mathrm{V}$ basis by the identity KPT scattering matrix

$$
\mathbf{S}=\left[\begin{array}{ll}
1 & 0 \\
0 & 1
\end{array}\right]
$$

The utility of SNPT can be seen by examining various polarization response plots. A polarization response plot shows a radar measureable as a function of incident tilt and ellipticity angle [2], [4]. For example, the SNPT copolar and crosspolar power polarization responses of a specular scatterer are flat planes at unity and zero, respectively. Compare this to the corresponding copolar power polarization responses for KPT and optic polarimetry which vary between unity and zero (see [2], [5]). Therefore, deviations in the SNPT polarization responses from these two reference planes are directly attributable to those features of the scatterer which are different from a specular scatterer. Because of this, we say that a specular reflector becomes the reference scatterer for SNPT.

We next illustrate the effects of modeling the radar on copolar differential phase defined as $\delta_{\mathrm{co}}^{K}(\phi, \epsilon)=\arg \left\{S_{22}(\phi, \epsilon) S_{11}^{*}(\phi, \epsilon)\right\}$ for $\mathrm{KPT}$ and as $\delta_{\mathrm{co}}^{S}(\phi, \epsilon)=\arg \left\{\ddot{S}_{22}(\phi, \epsilon) \ddot{S}_{11}^{*}(\phi, \epsilon)\right\}$ for SNPT. In [5] the copolar differential phase polarization response is shown for a scatterer modeled in the RAC in the $\mathrm{H}-\mathrm{V}$ basis as

$$
\begin{aligned}
\mathbf{S} & =\ddot{\mathbf{S}} \\
& =\left[\begin{array}{ll}
1 & 0 \\
0 & 2
\end{array}\right] .
\end{aligned}
$$

This can be thought of as modeling a small oblate raindrop that is in the Rayleigh scattering regime: the scatterer is much smaller than the wavelength and therefore not only is the copolar differential phase zero, but also there is zero phase shift upon backscatter for both vertical and horizontal incident polarizations. Physically, it does not matter what elliptical polarization is transmitted, the backscattered wave suffers no phase shift due to the particle at backscatter. Therefore, the copolar differential phase should be zero for all polarization bases. However, Fig. 13 in [5] shows that $\delta_{\text {co }}^{K}(\phi, \epsilon)$ for a scatterer modeled by (17) is in fact not identically zero. This superfluous nonzero differential phase is not due to the scatterer but rather due to the nature of the KPT basis transformation. In contrast, $\delta_{\mathrm{co}}^{S}(\phi, \epsilon)$ for this scatterer is always zero in any polarization basis as shown in [5].

For further insight into the reason that this superfluous differential phase occurs, consider $45^{\circ}$ linear incident polarization which can be decomposed into identical $\mathrm{H}$ and $\mathrm{V}$ electric field components. When a $45^{\circ}$ linear polarized wave is specularly reflected, the $\mathrm{H}$ and $\mathrm{V}$ components remain the same as the incident $\mathrm{H}$ and $\mathrm{V}$ components in the RAC, i.e., (9) is true, and the return is completely received by the KPT copolar channel. Let a differential phase shift now occur between the incident $\mathrm{H}$ and $\mathrm{V}$ components. Again the reflected $\mathrm{H}$ and $\mathrm{V}$ wave components remain the same as the incident $\mathrm{H}$ and $\mathrm{V}$ components, i.e., (9) remains true. However, the reflected wave will now be divided between the KPT copolar and cross-polar channels until the differential phase between the incident $\mathrm{H}$ and $\mathrm{V}$ components is $90^{\circ}$ whereby the reflected wave will be completely received by the KPT cross-polar channel. It is the modeling of the radar receive network that results in the particular definition of copolar used in KPT that causes the transference of power from copolar to cross-polar as the ellipticity angle of the incident wave goes from zero to $90^{\circ}$ (i.e., from llinear $45^{\circ}$ to circular). Coupled to the transference of power process is the occurence of the superfluous $\delta_{\mathrm{co}}^{K}(\phi, \epsilon)$. One could argue that an incident right circularly polarized wave actually does become left hand circular upon specular reflection and therefore the reflected wave polarization is in fact orthogonal to the incident polarization state. However, we feel that for the study of scatterers it is more appropriate and insightful to focus on the nature of the reflected field components, i.e., (9) is fundamental in defining copolar and eigenpolarizations. Note that optic polarimetrists could analogously argue that an incident $45^{\circ}$ linear polarized wave that is specularly reflected actually does become $-45^{\circ}$ linear polarized and therefore the reflected wave polarization state is orthogonal to the incident wave polarization state. Hence, the reflected wave should be directed into the cross-polar channel but is instead is called copolar in KPT! Again, SNPT circumvents these issues by considering (9) fundamental in defining the copolar reception state and eigenpolarizations and doing so eliminates the effects of modeling the radar and of reversal of propagation direction on the polarization signatures of scatterers.

\section{COVARIANCE MATRIX FOR SNPT}

Following [10] and [11], a covariance matrix can be used to describe incoherent scattering for SNPT. To construct the 
covariance matrix the outer product of the feature vector

$$
\boldsymbol{\Omega}^{T}=\left[\begin{array}{llll}
S_{\mathrm{HH}} & S_{\mathrm{VH}} & S_{\mathrm{HV}} & S_{\mathrm{VV}}
\end{array}\right]
$$

is taken which gives the covariance matrix in the $\mathrm{H}-\mathrm{V}$ basis (19) as shown at the bottom of the page where $\langle\cdot\rangle$ denotes ensemble averages. Note that the covariance matrix is Hermitian. Initially reciprocity is not invoked in order to maintain generality. Transformation of the covariance matrix to other polarization bases is accomplished by a unitary similarity transformation

$$
\boldsymbol{\Sigma}(\chi)=\mathbf{T}(\chi) \boldsymbol{\Sigma}_{0} \mathbf{T}^{-1}(\chi)
$$

where

$$
\mathbf{T}(\chi)=\Gamma^{2}\left[\begin{array}{cccc}
1 & \chi^{*} & \chi & \chi \chi^{*} \\
-\varrho^{2} \chi & \varrho^{2} & -\varrho^{2} \chi^{2} & \varrho^{2} \chi \\
-\varrho^{* 2} \chi^{*} & -\varrho^{* 2} \chi^{* 2} & \varrho^{* 2} & \varrho^{* 2} \chi^{*} \\
\chi \chi^{*} & -\chi^{*} & -\chi & 1
\end{array}\right]
$$

The individual members of the specular covariance matrix in (20) are represented as $R_{a b}, a, b=1,2,3,4$.

\section{A. Conditions for Optimum Polarizations}

Following [11], the optimum polarizations can be found in terms of the covariance functions themselves by taking the derivative of the covariance matrix with respect to $\chi^{*}$ and equating to zero. The derivative can be calculated as

$$
\frac{\partial \boldsymbol{\Sigma}(\chi)}{\partial \chi^{*}}=\mathbf{T}_{\mathbf{D}}(\chi) \boldsymbol{\Sigma}(\chi)-\boldsymbol{\Sigma}(\chi) \mathbf{T}_{\mathbf{D}}(\chi)
$$

where

$$
\mathbf{T}_{\mathbf{D}}(\chi)=\frac{\partial \mathbf{T}(\chi)}{\partial \chi^{*}} \mathbf{T}^{-1}(\chi)
$$

The conditions for copolar optimum polarizations are

$$
\begin{aligned}
\frac{\partial R_{11}}{\partial \chi^{*}} & =\Gamma\left[R_{21}+R_{13}\right] \\
& =\Gamma\left[\left\langle\ddot{S}_{21} \ddot{S}_{11}^{*}\right\rangle+\left\langle\ddot{S}_{11} \ddot{S}_{12}^{*}\right\rangle\right] \\
& =0 \\
\frac{\partial R_{44}}{\partial \chi^{*}} & =-\Gamma\left[R_{24}+R_{43}\right] \\
& =-\Gamma\left[\left\langle\ddot{S}_{21} \ddot{S}_{22}^{*}\right\rangle+\left\langle\ddot{S}_{22} \ddot{S}_{12}^{*}\right\rangle\right] \\
& =0
\end{aligned}
$$

and for cross-polar optimum polarizations

$$
\begin{aligned}
\frac{\partial R_{22}}{\partial \chi^{*}} & =\Gamma\left[R_{24}-R_{21}\right] \\
& =\Gamma\left[\left\langle\ddot{S}_{21} \ddot{S}_{22}^{*}\right\rangle-\left\langle\ddot{S}_{21} \ddot{S}_{11}^{*}\right\rangle\right] \\
& =0
\end{aligned}
$$

$$
\begin{aligned}
\frac{\partial R_{33}}{\partial \chi^{*}} & =\Gamma\left[R_{43}-R_{13}\right] \\
& =\Gamma\left[\left\langle\ddot{S}_{22} \ddot{S}_{12}^{*}\right\rangle-\left\langle\ddot{S}_{11} \ddot{S}_{12}^{*}\right\rangle\right] \\
& =0 .
\end{aligned}
$$

Thus optimum polarizations depend on interchannel covariances. For comparison, the conditions for optimum polarizations as given by [11] for KPT are listed next: for cross-polar optimum polarizations

$$
\left\langle S_{12} S_{22}^{*}\right\rangle-\left\langle S_{11} S_{12}^{*}\right\rangle=0
$$

and for copolar optimum polarizations

$$
\left\langle S_{11} S_{12}^{*}\right\rangle=0
$$

\section{B. Reciprocal Relations}

It can be shown that

$$
\begin{aligned}
& \ddot{S}_{11}\left(\frac{-1}{\chi^{*}}\right)=\ddot{S}_{22}(\chi) \\
& \ddot{S}_{22}\left(\frac{-1}{\chi^{*}}\right)=\ddot{S}_{11}(\chi) \\
& \ddot{S}_{12}\left(\frac{-1}{\chi^{*}}\right)=-\ddot{S}_{21}(\chi) \\
& \ddot{S}_{21}\left(\frac{-1}{\chi^{*}}\right)=-\ddot{S}_{12}(\chi)
\end{aligned}
$$

where $-1 / \chi^{*}$ is the orthogonal polarization to $\chi$. Using (30)-(33) in (20) it can be shown that

$$
\Sigma\left(\frac{-1}{\chi^{*}}\right)=\left[\begin{array}{rrrr}
R_{44} & -R_{34}^{*} & -R_{24}^{*} & R_{14}^{*} \\
-R_{34} & R_{33} & R_{23}^{*} & -R_{13}^{*} \\
-R_{24} & R_{23} & R_{22} & -R_{12}^{*} \\
R_{14} & -R_{13} & -R_{12} & R_{11}
\end{array}\right]
$$

from which the orthogonal power relations are found as

$$
\begin{aligned}
R_{11}\left(\frac{-1}{\chi^{*}}\right) & =P_{\mathrm{co}}^{A}\left(\frac{-1}{\chi^{*}}\right) \\
& =P_{\mathrm{co}}^{B}(\chi) \\
& =R_{44}(\chi) \\
R_{22}\left(\frac{-1}{\chi^{*}}\right) & =P_{x}^{A}\left(\frac{-1}{\chi^{*}}\right) \\
& =P_{x}^{B}(\chi) \\
& =R_{33}(\chi)
\end{aligned}
$$

where $P_{\mathrm{co}}^{A}(\chi)=R_{11}(\chi)$ and $P_{x}^{A}(\chi)=R_{22}(\chi)$.

$$
\boldsymbol{\Sigma}_{0}=\left\langle\boldsymbol{\Omega} \boldsymbol{\Omega}^{\dagger}\right\rangle=\left[\begin{array}{cccc}
\left\langle\left|S_{\mathrm{HH}}\right|^{2}\right\rangle & \left\langle S_{\mathrm{HH}} S_{\mathrm{VH}}^{*}\right\rangle & \left\langle S_{\mathrm{HH}} S_{\mathrm{HV}}^{*}\right\rangle & \left\langle S_{\mathrm{HH}} S_{\mathrm{VV}}^{*}\right\rangle \\
\left\langle S_{\mathrm{VH}} S_{\mathrm{HH}}^{*}\right\rangle & \left\langle\left|S_{\mathrm{VH}}\right|^{2}\right\rangle & \left\langle S_{\mathrm{VH}} S_{\mathrm{HV}}^{*}\right\rangle & \left\langle S_{\mathrm{VH}} S_{\mathrm{VV}}^{*}\right\rangle \\
\left\langle S_{\mathrm{HV}} S_{\mathrm{HH}}^{*}\right\rangle & \left\langle S_{\mathrm{HV}} S_{\mathrm{VH}}^{*}\right\rangle & \left\langle\left|S_{\mathrm{HVV}}\right|^{2}\right\rangle & \left\langle S_{\mathrm{HV}} S_{\mathrm{VV}}^{*}\right\rangle \\
\left\langle S_{\mathrm{VV}} S_{\mathrm{HH}}^{*}\right\rangle & \left\langle S_{\mathrm{VV}} S_{\mathrm{VH}}^{*}\right\rangle & \left\langle S_{\mathrm{VV}} S_{\mathrm{HV}}^{*}\right\rangle & \left\langle\left|S_{\mathrm{VV}}\right|^{2}\right\rangle
\end{array}\right]
$$




\section{Copolar Optimum Polarizations}

Optimum polarizations can be found using real linear algebra. The copolar power function, $R_{11}(\chi)$ can be expressed as

$$
R_{11}(\chi)=\mathbf{z}_{c}^{\dagger}(\chi) \mathbf{\Sigma}_{0} \mathbf{z}_{c}(\chi)
$$

where

$$
\mathbf{z}_{c}^{\dagger}(\chi)=\Gamma^{2}\left[\begin{array}{llll}
1 & \chi^{*} & \chi & \chi \chi^{*}
\end{array}\right]
$$

In order to find the optimum polarizations, the complex vector (38) is transformed to a real vector by

$$
\mathbf{y}(\chi)=\mathbf{Q} \mathbf{z}_{c}(\chi)
$$

where $2^{-1 / 2} \mathbf{Q}$ is unitary and $\mathrm{Q}$ is defined as

$$
\mathbf{Q}=\left[\begin{array}{rrrr}
1 & 0 & 0 & 1 \\
1 & 0 & 0 & -1 \\
0 & 1 & 1 & 0 \\
0 & -j & j & 0
\end{array}\right]
$$

Then $\mathbf{y}(\chi)$ takes the form

$$
\mathbf{y}^{T}(\chi)=\Gamma^{2}\left[1+\chi \chi^{*} \quad 1-\chi \chi^{*} \quad 2 \Re\{\chi\} \quad 2 \Im\{\chi\}\right]
$$

so that

$$
\mathbf{y}^{T} \mathbf{y}=2
$$

$\Re$ and $\Im$ specify the real and imaginary parts, respectively. Since the first member of the vector $y$ is unity, this allows the copolar power function to be written in the inhomogeneous Hermitian quadratic form

$$
R_{11}=\frac{1}{4}\left[\mathbf{v}^{T} \mathbf{W} \mathbf{v}+\mathbf{w}^{T} \mathbf{v}\right]
$$

where $\mathbf{W}$ is real and symmetric, and $\mathbf{v}$ is the real vector

$$
\mathbf{v}^{T}(\chi)=\Gamma^{2}\left[1-\chi \chi^{*} \quad 2 \Re\{\chi\} \quad 2 \Im\{\chi\}\right]
$$

and imposes the constraint

$$
\mathbf{v}^{\mathbf{T}} \mathbf{v}=1
$$

The members of $\mathbf{W}$ and $\mathbf{w}$ are

$$
\begin{aligned}
W_{11}= & 2\left(\left\langle\left|S_{\mathrm{HH}}\right|^{2}\right\rangle+\left\langle\left|S_{\mathrm{VV}}\right|^{2}\right\rangle\right) \\
W_{12}= & \Re\left\{\left\langle S_{\mathrm{HH}} S_{\mathrm{HV}}^{*}\right\rangle+\left\langle S_{\mathrm{HH}} S_{\mathrm{VH}}^{*}\right\rangle-\left\langle S_{\mathrm{VV}} S_{\mathrm{HV}}^{*}\right\rangle\right. \\
& \left.-\left\langle S_{\mathrm{VV}} S_{\mathrm{VH}}^{*}\right\rangle\right\} \\
W_{13}= & -\Im\left\{\left\langle S_{\mathrm{HH}} S_{\mathrm{VH}}^{*}\right\rangle-\left\langle S_{\mathrm{HH}} S_{\mathrm{HV}}^{*}\right\rangle+\left\langle S_{\mathrm{VV}} S_{\mathrm{HV}}^{*}\right\rangle\right. \\
& \left.-\left\langle S_{\mathrm{VV}} S_{\mathrm{VH}}^{*}\right\rangle\right\}
\end{aligned}
$$

$$
\begin{aligned}
W_{22}= & \left\langle\left|S_{\mathrm{HH}}\right|^{2}\right\rangle+\left\langle\left|S_{\mathrm{VV}}\right|^{2}\right\rangle+\left\langle\left|S_{\mathrm{HV}}\right|^{2}\right\rangle+\left\langle\left|S_{\mathrm{VH}}\right|^{2}\right\rangle \\
& +2 \Re\left\{\left\langle S_{\mathrm{HV}} S_{\mathrm{VH}}^{*}\right\rangle\right\}+2 \Re\left\{\left\langle S_{\mathrm{HH}} S_{\mathrm{VV}}^{*}\right\rangle\right\} \\
W_{23}= & \Im\left\{S_{\mathrm{VH}} S_{\mathrm{HV}}^{*}\right\} \\
W_{33}= & \left\langle\left|S_{\mathrm{HH}}\right|^{2}\right\rangle+\left\langle\left|S_{\mathrm{VV}}\right|^{2}\right\rangle+\left\langle\left|S_{\mathrm{HV}}\right|^{2}\right\rangle+\left\langle\left|S_{\mathrm{VH}}\right|^{2}\right\rangle \\
& +2 \Re\left\{\left\langle S_{\mathrm{HH}} S_{\mathrm{VV}}^{*}\right\rangle\right\}-2 \Re\left\{\left\langle S_{\mathrm{HV}} S_{\mathrm{VH}}^{*}\right\rangle\right\}
\end{aligned}
$$

and (47), shown at the bottom of the page. Optimum polarizations can be found using Lagrange multipliers [18]. A sixth-order polynomial will result and thus, there are six possible optimum polarizations for the general case where $S_{\mathrm{HV}} \neq S_{\mathrm{VH}}$. The Lagrange multiplier method used here is similiar to that offered by [19] and [20].

Reciprocity is now invoked, i.e., $S_{\mathrm{HV}}=S_{\mathrm{VH}}$ which simplifies $\mathbf{W}$ and $\mathbf{w}$ :

$$
\begin{aligned}
W_{11}= & 2\left(\left\langle\left|S_{\mathrm{HH}}\right|^{2}\right\rangle+\left\langle\left|S_{\mathrm{VV}}\right|^{2}\right\rangle\right) \\
W_{12}= & 2 \Re\left\{\left\langle S_{\mathrm{HH}} S_{\mathrm{HV}}^{*}\right\rangle-\left\langle S_{\mathrm{VV}} S_{\mathrm{HV}}^{*}\right\rangle\right\} \\
W_{13}= & 0 \\
W_{22}= & \left\langle\left|S_{\mathrm{HH}}\right|^{2}\right\rangle+\left\langle\left|S_{\mathrm{VV}}\right|^{2}\right\rangle+4\left\langle\left|S_{\mathrm{HV}}\right|^{2}\right\rangle \\
& \quad+2 \Re\left\{\left\langle S_{\mathrm{HH}} S_{\mathrm{VV}}^{*}\right\rangle\right\} \\
W_{23}= & 0 \\
W_{33}= & \left\langle\left|S_{\mathrm{HH}}\right|^{2}\right\rangle+\left\langle\left|S_{\mathrm{VV}}\right|^{2}\right\rangle+2 \Re\left\{\left\langle S_{\mathrm{HH}} S_{\mathrm{VV}}^{*}\right\rangle\right\}
\end{aligned}
$$

and (49), shown at the bottom of the page. The method of Lagrange multipliers can be applied to (43) which results in the following set of linear equations:

$$
\mathbf{W v}-\lambda \mathbf{v}=-\frac{1}{2} \mathbf{w}
$$

with the constraint imposed by (45) where $\lambda$ is the Lagrange multiplier. Because of the zero entries in (48) and (49), the solution is simplified. The linear equation in (50) involving the imaginary part of $\chi, v_{3}$, is

$$
v_{3}\left(W_{33}-\lambda\right)=0
$$

where $v_{1}, v_{2}$, and $v_{3}$ are the individual members of $\mathbf{v}$. Thus, either $v_{3}=0$, i.e., the $\Im\{\chi\}=0$, or $\lambda=W_{33}$. For nonlinear optimum polarizations to exist $\lambda=W_{33}$. Using this value for the Lagrange mutiplier, the remaining system of equations may be solved analytically for $v_{1}$ and $v_{2}$. However, the constraint (45) must be checked for a valid nonlinear polarization solution to exist. The remaining optimum polarizations are found by setting $v_{3}=0$ and solving the resulting reduced set of linear equations with the constraint $v_{1}^{2}+v_{2}^{2}=1$. A fourth order polynomial in $\lambda$ will result and thus there are four possible linear optimum polarizations. In comparison,

$$
\mathbf{w}=\left[\begin{array}{c}
2\left(\left\langle\left. S_{\mathrm{HH}}\right|^{2}-\left\langle\left|S_{\mathrm{VV}}\right|^{2}\right\rangle\right)\right. \\
2 \Re\left\{\left\langle S_{\mathrm{HH}} S_{\mathrm{HV}}^{*}\right\rangle+\left\langle S_{\mathrm{HH}} S_{\mathrm{VH}}^{*}\right\rangle+\left\langle S_{\mathrm{VV}} S_{\mathrm{HV}}^{*}\right\rangle-\left\langle S_{\mathrm{VV}} S_{\mathrm{VH}}^{*}\right\rangle\right\} \\
2 \Im\left\{\left\langle S_{\mathrm{HH}} S_{\mathrm{HV}}^{*}\right\rangle-\left\langle S_{\mathrm{HH}} S_{\mathrm{VH}}^{*}\right\rangle+\left\langle S_{\mathrm{VV}} S_{\mathrm{HV}}^{*}\right\rangle-\left\langle S_{\mathrm{VV}} S_{\mathrm{VH}}^{*}\right\rangle\right\}
\end{array}\right]
$$

$$
\mathbf{w}^{T}=\left[2\left(\left\langle\left|S_{\mathrm{HH}}\right|^{2}\right\rangle-\left\langle\left|S_{\mathrm{VV}}\right|^{2}\right\rangle\right) \quad 4 \Re\left\{\left\langle S_{\mathrm{HH}} S_{\mathrm{HV}}^{*}\right\rangle+\left\langle S_{\mathrm{VV}} S_{\mathrm{HV}}^{*}\right\rangle\right\} \quad 0\right]
$$


there are six possible KPT copolar optimum polarizations all of which can be elliptical [11].

\section{Cross-Polar Optimum Polarizations}

The cross-polar power function, $R_{22}(\chi)$, can be expressed as

$$
R_{22}(\chi)=\mathbf{z}_{x}^{\dagger}(\chi) \mathbf{\Sigma}_{0} \mathbf{z}_{x}(\chi)
$$

where

$$
\mathbf{z}_{x}^{\dagger}=\Gamma^{2}\left[\begin{array}{llll}
-\chi & 1 & -\chi^{2} & \chi
\end{array}\right]
$$

The cross-polar power can be expressed in terms of the inhomogeneous Hermitian quadratic form

$$
R_{22}=\mathbf{v}^{T} \mathbf{B v}+\mathbf{b}^{T} \mathbf{v}
$$

where $\mathbf{B}$ is real and symmetric and $\mathbf{v}$ is given by (44). By expanding (52) and (54) and equating coefficients, $\mathbf{B}$ and $\mathrm{b}$ are found to be

$$
\begin{aligned}
B_{11}= & 0.5\left[\left\langle\left|S_{\mathrm{HV}}\right|^{2}\right\rangle+\left\langle\left|S_{\mathrm{VH}}\right|^{2}\right\rangle\right] \\
B_{12}= & 0.25 \Re\left\{\left\langle S_{\mathrm{VV}} S_{\mathrm{VH}}^{*}\right\rangle-\left\langle S_{\mathrm{HH}} S_{\mathrm{VH}}^{*}\right\rangle\right. \\
& \left.-\left\langle S_{\mathrm{HH}} S_{\mathrm{HV}}^{*}\right\rangle+\left\langle S_{\mathrm{VV}} S_{\mathrm{HV}}^{*}\right\rangle\right\} \\
B_{13}= & 0.25 \Im\left\{\left\langle S_{\mathrm{VV}} S_{\mathrm{HV}}^{*}\right\rangle-\left\langle S_{\mathrm{HH}} S_{\mathrm{HV}}^{*}\right\rangle\right. \\
& \left.-\left\langle S_{\mathrm{VV}} S_{\mathrm{VH}}^{*}\right\rangle+\left\langle S_{\mathrm{HH}} S_{\mathrm{VH}}^{*}\right\rangle\right\} \\
B_{22}= & 0.25\left[\left\langle\left|S_{\mathrm{HH}}\right|^{2}\right\rangle+\left\langle\left|S_{\mathrm{VV}}\right|^{2}\right\rangle+\left\langle\left|S_{\mathrm{HV}}\right|^{2}\right\rangle\right. \\
& \left.+\left\langle\left|S_{\mathrm{VH}}\right|^{2}\right\rangle-2 \Re\left\{\left\langle S_{\mathrm{HV}} S_{\mathrm{VH}}^{*}\right\rangle+\left\langle S_{\mathrm{HH}} S_{\mathrm{VV}}^{*}\right\rangle\right\}\right] \\
B_{23}= & 0.5 \Im\left\{\left\langle S_{\mathrm{HV}} S_{\mathrm{VH}}^{*}\right\rangle\right\} \\
B_{33}= & 0.25\left[\left\langle\left|S_{\mathrm{HH}}\right|^{2}\right\rangle+\left\langle\left|S_{\mathrm{VV}}\right|^{2}\right\rangle+\left\langle\left|S_{\mathrm{HV}}\right|^{2}\right\rangle+\left\langle\left|S_{\mathrm{VH}}\right|^{2}\right\rangle\right. \\
& \left.+2 \Re\left\{\left\langle S_{\mathrm{HV}} S_{\mathrm{VH}}^{*}\right\rangle-\left\langle S_{\mathrm{HH}} S_{\mathrm{VV}}^{*}\right\rangle\right\}\right]
\end{aligned}
$$

and (56), shown at the bottom of the page. The extrema of (54) can again be found by applying the method of Lagrange multipliers. There will be in general six optimum polarizations for the nonreciprocal case. Invoking reciprocity where $S_{\mathrm{HV}}=$ $S_{\mathrm{VH}},(55)$ and (56) simplify to

$$
\begin{aligned}
& B_{11}=\left\langle\left|S_{\mathrm{HV}}\right|^{2}\right\rangle \\
& B_{12}=0.5 \Re\left\{\left\langle S_{\mathrm{VV}} S_{\mathrm{HV}}^{*}\right\rangle-\left\langle S_{\mathrm{HH}} S_{\mathrm{HV}}^{*}\right\rangle\right\} \\
& B_{13}=0 \\
& B_{22}=0.25\left[\left\langle\left|S_{\mathrm{HH}}\right|^{2}\right\rangle+\left\langle\left|S_{\mathrm{VV}}\right|^{2}\right\rangle-2 \Re\left\{\left\langle S_{\mathrm{HH}} S_{\mathrm{VV}}^{*}\right\rangle\right\}\right] \\
& B_{23}=0 \\
& B_{33}=0.25\left[\left\langle\left|S_{\mathrm{HH}}\right|^{2}\right\rangle+\left\langle\left|S_{\mathrm{VV}}\right|^{2}\right\rangle+4\left\langle\left|S_{\mathrm{HV}}\right|^{2}\right\rangle\right. \\
& \left.-2 \Re\left\{\left\langle S_{\mathrm{HH}} S_{\mathrm{VV}}^{*}\right\rangle\right\}\right]
\end{aligned}
$$

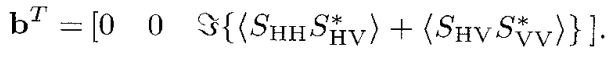

Because of the zero entries, the extrema problem is simplified. Upon applying the method of Lagrange multipliers, the system of linear equations is found to be

$$
\begin{array}{r}
v_{1}\left(B_{11}-\lambda\right)+v_{2} B_{12}=0 \\
v_{1} B_{12}+v_{2}\left(B_{22}-\lambda\right)=0 \\
2 v_{3}\left(B_{33}-\lambda\right)+b_{3}=0
\end{array}
$$

with the constraint imposed by (45) where $b_{3}$ is the third member of the vector $b$. One solution to this set of equations is $v_{1}=v_{2}=0, v_{3}=1$ which corresponds to circular polarizations. Thus, circular polarizations are always optimum polarizations. The other solutions are found from the Lagrange multipliers satisfying

$$
\lambda^{2}-\left(B_{11}+B_{22}\right) \lambda+B_{11} B_{22}-B_{12}^{2}=0
$$

and then $\mathbf{v}$ is found from

$$
\begin{aligned}
& v_{3}=-\frac{b_{3}}{2\left(B_{33}-\lambda\right)} \\
& v_{2}^{2}=\frac{\left(1-v_{3}^{2}\right)\left(B_{11}-\lambda\right)^{2}}{B_{12}^{2}+\left(B_{11}-\lambda\right)^{2}} \\
& v_{1}^{2}=1-v_{2}^{2}-v_{3}^{2} .
\end{aligned}
$$

The $\chi$ can be found from $\mathbf{v}$ via (44). Thus, besides circular polarization, there are possibly two other optimum polarizations (and their orthogonal complements) which will be linear if $b_{3}$ is zero. This is an important condition to recognize: the cross-polar power minimum polarization state will be linear if:

$$
\Im\left\{\left\langle S_{\mathrm{HH}} S_{\mathrm{HV}}^{*}\right\rangle\right\}=-\Im\left\{\left\langle S_{\mathrm{HV}} S_{\mathrm{VV}}^{*}\right\rangle\right\}
$$

for reciprocal scattering. This condition does not apply to KPT. In KPT there are also three orthogonal pairs of optimum polarizations but they are, in general, elliptical. The polarization state for which the cross-polar power attains an absolute minimum is referred to as the characteristic polarization state.

We finally note that the equations developed above are based on the SNPT change of basis (12) which is for backscatter; however, since this change of basis expression is identical to the change of basis equation for forward scattering

$$
\tilde{\mathbf{S}}=\mathbf{U}^{-1}\left[\begin{array}{ll}
S_{\mathrm{HH}}^{f s} & S_{\mathrm{HV}}^{f s} \\
S_{\mathrm{VH}}^{f s} & S_{\mathrm{VV}}^{f s}
\end{array}\right] \mathbf{U},
$$

except that the entries of $\mathbf{S}$ are now forward scattering amplitudes, the above equations are applicable to other forward and bistatic scattering problems as well.

\section{Modeling Precipitation Particles}

The optimum polarizations for ensembles of scatterers are now calculated from simulated covariance matrices. The model used is described in [21] which employs the transition ( $T-$ matrix method to calculate the $2 \times 2$ scattering matrix and then integrates over the specified size and orientation distributions.

$$
\mathbf{b}=\left[\begin{array}{c}
0.5\left(\left\langle\left|S_{\mathrm{VH}}\right|^{2}-\left\langle\left|S_{\mathrm{HV}}\right|^{2}\right\rangle\right)\right. \\
0.5 \Re\left\{\left\langle S_{\mathrm{VV}} S_{\mathrm{VH}}^{*}\right\rangle-\left\langle S_{\mathrm{HH}} S_{\mathrm{VH}}^{*}\right\rangle+\left\langle S_{\mathrm{HH}} S_{\mathrm{HV}}^{*}\right\rangle-\left\langle S_{\mathrm{VV}} S_{\mathrm{HV}}^{*}\right\rangle\right\} \\
0.5 \Im\left\{\left\langle S_{\mathrm{HH}} S_{\mathrm{HV}}^{*}\right\rangle-\left\langle S_{\mathrm{VV}} S_{\mathrm{HV}}^{*}\right\rangle-\left\langle S_{\mathrm{VV}} S_{\mathrm{VH}}^{*}\right\rangle+\left\langle S_{\mathrm{HH}} S_{\mathrm{VH}}^{*}\right\rangle\right\}
\end{array}\right] z
$$


TABLE I

The Optimum Polarizations For the Ensemble of Scatterers Described by tHe Covariance Matrix in (69)

\begin{tabular}{|c|c|c|c|c|c|}
\hline \multicolumn{6}{|c|}{ OPTIMUM POLARIZATIONS } \\
\hline & & \multicolumn{2}{|c|}{ KPT } & \multicolumn{2}{|c|}{ SNPT } \\
\hline & & tilt & ellip. & tilt & ellip. \\
\hline \multirow{4}{*}{ COPOL } & max. & $0^{\circ}$ & $0^{\circ}$ & $0^{\circ}$ & $0^{\circ}$ \\
\hline & saddle & $90^{\circ}$ & $0^{\circ}$ & $=$ & $=$ \\
\hline & $\min .1$ & $-84.6^{\circ}$ & $-38.4^{\circ}$ & $=$ & - \\
\hline & $\min .2$ & $84.6^{\circ}$ & $38.4^{\circ}$ & $90^{\circ}$ & $0^{\circ}$ \\
\hline \multirow{3}{*}{ XPOL } & $\max$. & $-45^{\circ}$ & $43.7^{\circ}$ & $0^{\circ}$ & $45^{\circ}$ \\
\hline & saddle & $45^{\circ}$ & $1.3^{\circ}$ & $45^{\circ}$ & $0^{\circ}$ \\
\hline & min. & $0^{\circ}$ & $0^{\circ}$ & $0^{\circ}$ & $0^{\circ}$ \\
\hline
\end{tabular}

The wavelength is $5.5 \mathrm{~cm}$. The model was modified so that arbitrary mean canting angles for ensembles of particles could be included. This was accomplished by using the Fisher distribution [22], [23] which is equivalent to a two dimensional Gaussian distribution that has been mapped to a sphere. For details of the Fisher distribution, see Appendix C.

An exponential size distribution of equilibrium shaped raindrops (oblate spheriods) is modeled as $N(D)=$ $N_{0} \exp \left\{-3.67\left(D / D_{0}\right)\right\}$ where $D$ is the diameter of an equi-volumetric spherical raindrop and $D_{0}$ is the median diameter. The parameters for the exponential distribution are: $N_{0}=8000 \mathrm{~mm}^{-1} \mathrm{~m}^{-3}, D_{0}=2.56 \mathrm{~mm}, D_{\min }=0.5 \mathrm{~mm}$, $D_{\max }=7 \mathrm{~mm}$. A mean canting angle in the polarization plane (plane perpendicular to the radar line of sight) and a canting angle variance is specified using the Fisher distribution. The Fisher distribution parameters are $\bar{\theta}=\bar{\phi}^{\prime}=0$ and $\kappa=100$ where $\bar{\theta}$ and $\bar{\phi}^{\prime}$ represent the mean canting angle in a spherical coordinate system with $\theta$ being the elevation angle and $\phi^{\prime}$ the azimuth. The incident wave is directed along the negative $x$ axis (see [21] for details). Thus for this orientation distribution the mean canting angle is zero (minor axis vertical) and the standard deviation as calculated by (C5) is approximately $8.7^{\circ}$. Because of reciprocity the covariance matrix used here will have the form [10]

$$
\boldsymbol{\Sigma}_{0}=\left[\begin{array}{ccc}
\left\langle\left|S_{\mathrm{HH}}\right|^{2}\right\rangle & \sqrt{2}\left\langle S_{\mathrm{HH}} S_{\mathrm{HV}}^{*}\right\rangle & \left\langle S_{\mathrm{HH}} S_{\mathrm{VV}}^{*}\right\rangle \\
\sqrt{2}\left\langle S_{\mathrm{HV}} S_{\mathrm{HH}}^{*}\right\rangle & 2\left\langle\left|S_{\mathrm{HV}}\right|^{2}\right\rangle & \sqrt{2}\left\langle S_{\mathrm{HV}} S_{\mathrm{VV}}^{*}\right\rangle \\
\left\langle S_{\mathrm{VV}} S_{\mathrm{HH}}^{*}\right\rangle & \sqrt{2}\left\langle S_{\mathrm{VV}} S_{\mathrm{HV}}^{*}\right\rangle & \left\langle\left|S_{\mathrm{VV}}\right|^{2}\right\rangle
\end{array}\right]
$$

The covariance matrix for the ensemble then is

$$
\boldsymbol{\Sigma}_{\mathbf{0}}=\left[\begin{array}{lll}
1.0 & 0.0 & 0.6183 e^{-j 5.04^{\circ}} \\
0.0 & 0.0036 & 0.0 \\
0.6183 e^{j 5.04^{\circ}} & 0.0 & 0.4119
\end{array}\right]
$$

where the matrix has been normalized by $\left\langle\left|S_{\mathrm{HH}}\right|^{2}\right\rangle$ with $10 \log \left[\left\langle\left|S_{\mathrm{HH}}\right|^{2}\right\rangle\right]=57.7 \mathrm{dBZ}$ which is conventional radar reflectivity. The electric fields are modeled with the $e^{j \omega t} e^{-j k r}$ time-space convention and therefore $\delta_{\mathrm{co}}$ is defined as $\arg \left\{\left\langle S_{\mathrm{VV}} S_{\mathrm{HH}}^{*}\right\rangle\right\}$. From the covariance matrix (69), $\left\langle S_{\mathrm{HH}} S_{\mathrm{HV}}^{*}\right\rangle=\left\langle S_{\mathrm{VV}} S_{\mathrm{HV}}^{*}\right\rangle=0$ which satisfies the conditions (24)-(29) which means that $\mathrm{H}$ and $\mathrm{V}$ are optimum copolar and cross-polar polarizations for both KPT and SNPT. All of the optimum polarizations are listed in Table I (orthogonal complements of the cross-polar optimum polarizations are not listed). The characteristic polarization, the cross-polar minimum, is horizontal.
The polarization response plots for this ensemble of scatterers are now compared for KPT and SNPT. Fig. 3 compares the copolar power $(Z)$, copolar differential reflectivity $\left(Z_{\mathrm{DR}}\right)$ and copolar differential phase $\left(\delta_{\mathrm{co}}\right)$ polarization responses while Fig. 4 compares the copolar correlation coefficient $\left(\rho_{\text {co }}\right)$ and the magnitude of the co-to-cross covariance $\left(\left|R_{x}\right|\right)$. The KPT polarization responses are found in the left panels while the corresponding SNPT polarization responses are found to the right. The generalized copolar correlation coefficient, calculated as

$$
\rho_{\mathrm{co}}=\frac{\left|R_{\mathrm{co}}\right|}{\left(P_{\mathrm{co}}^{A} P_{x}\right)^{1 / 2}},
$$

where $R_{\mathrm{co}}=\left\langle S_{22} S_{11}^{*}\right\rangle$ (or $R_{\mathrm{co}}=\left\langle\ddot{S}_{22} \ddot{S}_{11}^{*}\right\rangle$ for SNPT), is an important microphysical indicator of the melting level and of mixed precipitation types [24], [25]. The co-to-cross covariance is defined as $R_{x}^{K}=S_{11} S_{21}^{*}$ for KPT and as $R_{x}^{S}=\ddot{S}_{11} \ddot{S}_{21}^{*}$ for SNPT.

There are two KPT copolar power minima located at $\phi=$ $-84.6^{\circ}, \epsilon=-38.4^{\circ}$ and $\phi=84.6^{\circ}, \epsilon=38.4^{\circ}$. Neither of these KPT copolar minima polarization states are copolar optimum polarizations for SNPT as can be seen by comparing the KPT and SNPT copolar power plots. All of the KPT polarization response plots show minima or maxima in the vicinity of the KPT copolar power minima polarization states. These minima and maxima are caused by the radar receive network modeled in the KPT basis transformation as discussed previously. Since the minima and maxima for $Z_{\mathrm{DR}}^{K}(\phi, \epsilon)$ and $\delta_{\mathrm{co}}^{K}(\phi, \epsilon)$ are located at the copolar power minima, one could be mislead into believing that a greater sensitivity to these parameters might be achieved by making measurements in these or the surrounding elliptical polarization bases. The problem with such a scheme is seen by examining the KPT $\rho_{\mathrm{co}}$ plot which decreases in value around the copolar power minima and in fact $\rho_{\mathrm{co}}^{K}(\phi, \epsilon)$ does go to zero at the KPT copolar power minima. As $\rho_{\text {co }}(\phi, \epsilon)$ decreases, the fluctuations in $\delta_{\mathrm{co}}(\phi, \epsilon)$ and $Z_{\mathrm{DR}}(\phi, \epsilon)$ increase very rapidly due to Rayleigh fading [26]. This effect substantially reduces the accuracy to which $\delta_{\mathrm{co}}(\phi, \epsilon)$ and $Z_{\mathrm{DR}}(\phi, \epsilon)$ can be estimated with dual polarization radars.

Since the conditions for optimum polarizations depend on co-to-cross channel covariances, it is insightful to examine the magnitude of $R_{x}$ show in Fig. 4. The magnitude of the KPT $R_{x}$ appears to approach zero at seven locations (three are hidden). In accordance with (28), $\left|R_{x}^{K}\right|$ has only four nulls whoses locations are given by the KPT copolar optimum polarizations in Table I. Another null does occur at $\phi=-90^{\circ}, \epsilon=0^{\circ}$ but this represents the same polarization as $\phi=90^{\circ}, \epsilon=0^{\circ}$. There are no minima in $\left|R_{x}^{S}\right|$ at the ellipitical polarization states and these KPT $\left|R_{x}\right|$ minima at elliptical polarizations exist due to the modeling of the radar receive network in the KPT basis transformation. As a result, KPT theory yields more copolar optimum polarizations than does SNPT for this class of scatterers. We finally note that that maximum and minimum SNPT $\delta_{\mathrm{co}}(\phi, \epsilon)$ and $Z_{\mathrm{DR}}(\phi, \epsilon)$ occur at the characteristic polarization state or its orthogonal complement polarization state and that the SNPT $\rho_{\mathrm{co}}(\phi, \epsilon)$ is always greater than 0.96 . 

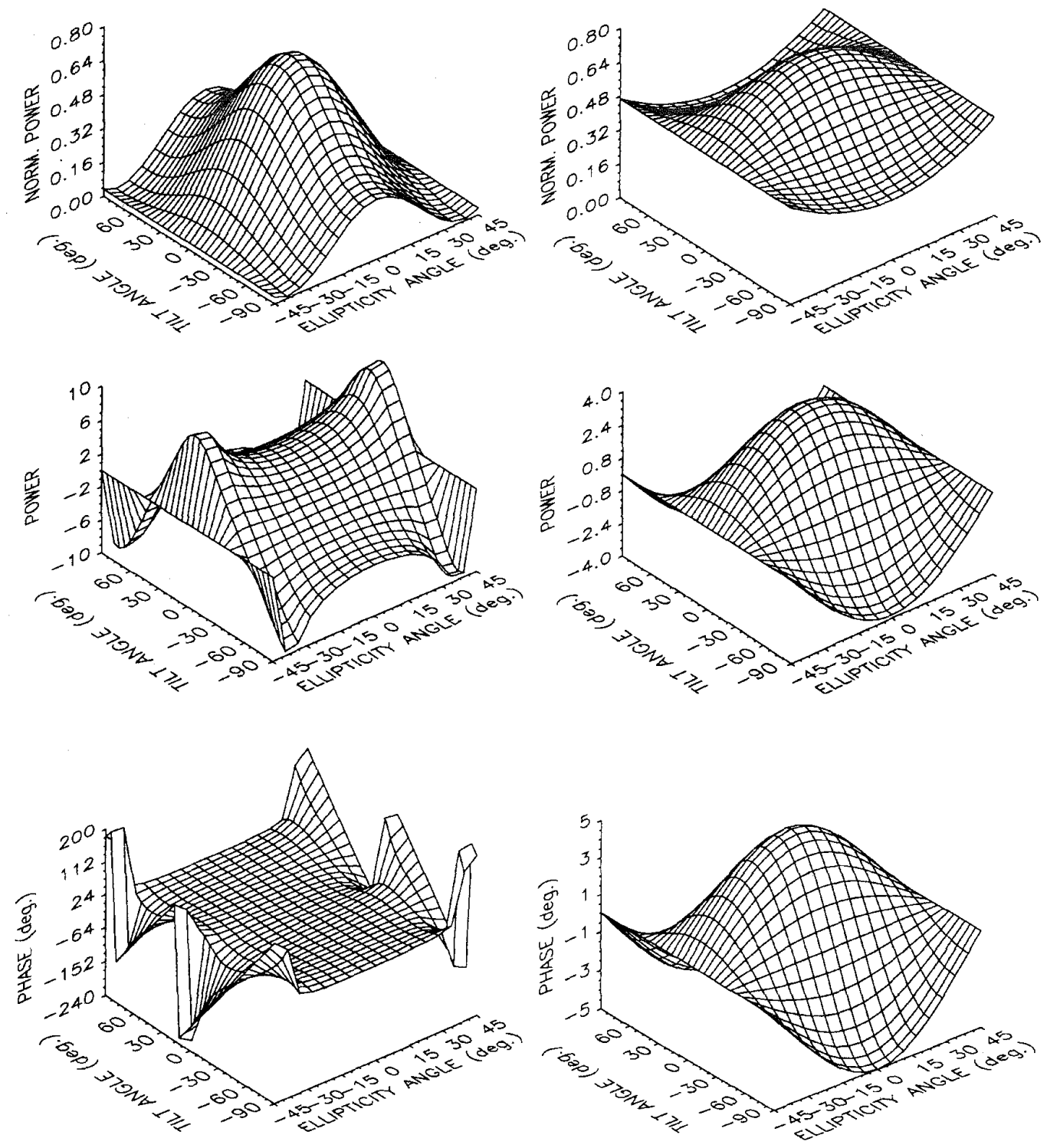

Fig. 3. Polarization response plots as functions of transmit tilt $(\phi)$ and ellipticity $(\epsilon)$ angles for the ensemble of raindrops described by (69). Left column is due to KPT and the right column is due to SNPT. From top to bottom: Copolar power, copolar differential power and copolar differential phase. Differential reflectivity is in decibel scale.

The effects of a nonzero mean canting angle is illustrated next by using the same size distribution of raindrops as described by (69) and letting $\bar{\theta}=20^{\circ}$ and $\bar{\phi}^{\prime}=90^{\circ}$ in the Fisher distribution. This gives a mean tilt angle of $20^{\circ}$ from vertical in the plane of polarization. For polarization definitions, a positive tilt angle is measured counterclockwise from the horizontal axis. Thus, the corresponding tilt angle is $-20^{\circ}$ for polarization definitions. The covariance matrix is

$$
\Sigma_{0}=\left[\begin{array}{lll}
1.0 & 0.1813 e^{j-171.4^{\circ}} & 0.6955 e^{j-3.76^{\circ}} \\
0.1813 e^{j 171.4^{\circ}} & 0.0431 & 0.1166 e^{j 166.6^{\circ}} \\
0.6955 e^{j 3.76^{\circ}} & 0.1166 e^{-j 166.6^{\circ}} & 0.5068
\end{array}\right]
$$

where the matrix is normalized by $\left\langle\left|S_{\mathrm{HH}}\right|^{2}\right\rangle$ with $10 \log \left[\left\langle\left|S_{\mathrm{HH}}\right|^{2}\right\rangle \mid=57.3 \mathrm{dBZ}\right.$. The optimum polarizations are given in Table II. Common optimum polarizations for KPT and SNPT are the copolar maximum and cross-polar minimum which are both linear at $-20^{\circ}$. This is the characteristic polarization state. Also, the SNPT copolar minimum corresponds with the copolar KPT saddle point. For SNPT the cross-polar maximum occurs (and always does) for circular polarizations but for KPT, the cross-polar maximum is at a tilt angle of $25.0^{\circ}$ and ellipticity angle of $43.7^{\circ}$. There are also two copolar minimums for KPT which are again not optimum polarizations in SNPT and are attributed to the radar receive network modeled in KPT. Note that the condition for SNPT linear characteristic polarization state, (66), is satisfied by the matrix in (71).

Next, the same particle size distribution is given a mean canting angle outside the polarization plane by letting $\bar{\theta}=20^{\circ}$ 

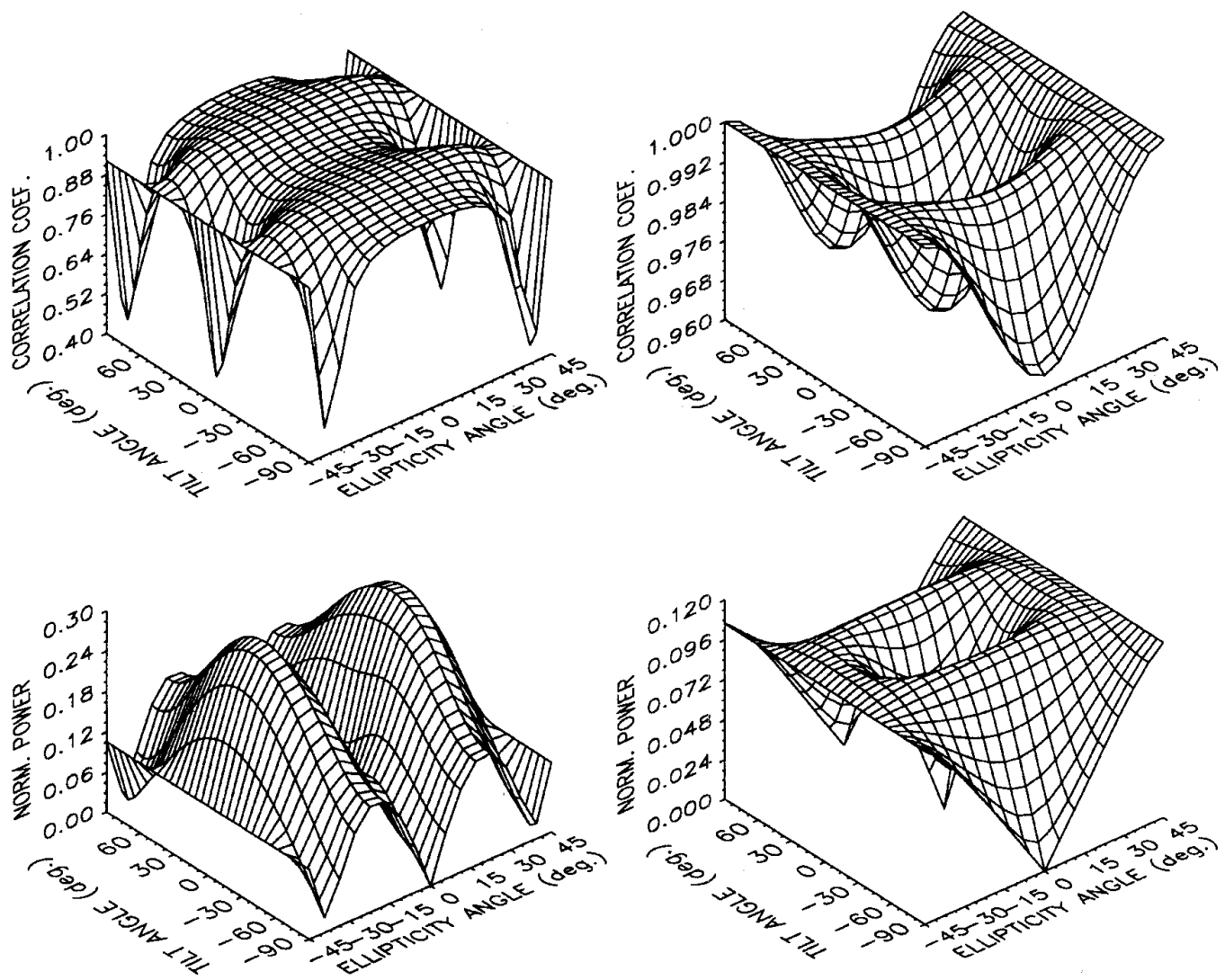

Fig. 4. Polarization response plots as functions of transmit tilt ( $\phi)$ and ellipticity ( $\epsilon$ ) angles for the ensemble of raindrops described by (69). Left column is due to KPT and the right column is due to SNPT. Top panels: Copolar correlation coefficient. Bottom panels: cross covariance.

TABLE II

The Optimum Polarizations for the Ensemble of SCATTERERS Described By THE Covariance Matrix in (71)

\begin{tabular}{|c|c|c|c|c|c|}
\hline \multicolumn{6}{|c|}{ OPTIMUM POLARIZATIONS } \\
\hline & & \multicolumn{2}{|c|}{ KPT } & \multicolumn{2}{|c|}{ SNPT } \\
\hline & & tilt & ellip. & tilt & ellip. \\
\hline \multirow{4}{*}{ COPOL } & $\max$. & $-20^{\circ}$ & $0^{\circ}$ & $-20^{\circ}$ & $0^{\circ}$ \\
\hline & saddle & $70.0^{\circ}$ & $0.0^{\circ}$ & - & 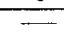 \\
\hline & $\min .1$ & $64.5^{\circ}$ & $38.5^{\circ}$ & - & 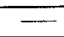 \\
\hline & min. 2 & $75.5^{\circ}$ & $-38.5^{\circ}$ & $70^{\circ}$ & $0^{\circ}$ \\
\hline \multirow{3}{*}{ XPOL } & $\max$ & $25.0^{\circ}$ & $43.7^{\circ}$ & $0^{\circ}$ & $45^{\circ}$ \\
\hline & saddle & $25.0^{\circ}$ & $1.3^{\circ}$ & $25^{\circ}$ & $0^{\circ}$ \\
\hline & min. & $-20^{\circ}$ & $0^{\circ}$ & $-20^{\circ}$ & $0^{\circ}$ \\
\hline
\end{tabular}

and $\bar{\phi}^{\prime}=50^{\circ}$. The covariance matrix is

$$
\boldsymbol{\Sigma}_{\mathbf{0}}=\left[\begin{array}{lll}
1.0 & 0.1371 e^{-j 171.8^{\circ}} & 0.6804 e^{-j 3.84^{\circ}} \\
0.1371 e^{j 171.8^{\circ}} & 0.026 & 0.0857 e^{j 166.9^{\circ}} \\
0.6804 e^{j 3.84^{\circ}} & 0.0857 e^{-j 166.9^{\circ}} & 0.4873
\end{array}\right]
$$

where the matrix is normalized by $\left\langle\left|S_{\mathrm{HH}}\right|^{2}\right\rangle$ with $10 \log \left[\left\langle\left|S_{\mathrm{HH}}\right|^{2}\right\rangle\right]=57.4 \mathrm{dBZ}$. The optimum polarizations are given in Table III. Common optimum polarizations for KPT and SNPT are again the copolar maximum and cross-polar minimum which are both linear $-15.6^{\circ}$. This angle can be obtained by projecting the mean canting angle onto the plane of polarization and is termed the apparent mean canting angle. The other common optimum polarizations are the KPT copolar saddle point and the SNPT copolar minimum. As
TABLE III

The Optimum Polarizations for the ENSEMBle of Scatterers Described by the Covariance MatriX IN (72)

\begin{tabular}{|l|l|r|r||r|r|}
\hline \multicolumn{6}{|c|}{ OPTIMUM POLARIZATIONS } \\
\hline \hline \multirow{2}{*}{} & \multicolumn{2}{|c|}{ KPT } & \multicolumn{2}{|c|}{ SNPT } \\
\cline { 2 - 6 } \multicolumn{2}{|c|}{ tilt } & \multicolumn{1}{|c|}{ ellip. } & tilt & ellip. \\
\hline \multirow{3}{*}{ COPOL } & max. & $-15.6^{\circ}$ & $0.0^{\circ}$ & $-15.6^{\circ}$ & $0^{\circ}$ \\
\cline { 2 - 6 } & saddle & $74.4^{\circ}$ & $0.0^{\circ}$ & - & - \\
\cline { 2 - 6 } & min. 1 & $79.7^{\circ}$ & $38.8^{\circ}$ & - & - \\
\cline { 2 - 6 } & min. 2 & $69.2^{\circ}$ & $38.8^{\circ}$ & $74.4^{\circ}$ & $0^{\circ}$ \\
\hline \multirow{3}{*}{ XPOL } & max. & $29.4^{\circ}$ & $43.9^{\circ}$ & $0^{\circ}$ & $45^{\circ}$ \\
\cline { 2 - 6 } & saddle & $29.4^{\circ}$ & $-1.1^{\circ}$ & $29.4^{\circ}$ & $0^{\circ}$ \\
\cline { 2 - 7 } & min. & $-15.6^{\circ}$ & $0^{\circ}$ & $-15.6^{\circ}$ & $0^{\circ}$ \\
\hline
\end{tabular}

before the the SNPT cross-polar saddle point is located $45^{\circ}$ in tilt angle away from the tilt angle of the cross-polar minimum.

The numerical method used here failed to locate the KPT copolar minimum polarizations even though double precision (32-b representation) was used. In order to find the correct values for the Lagrange multipliers, a simple search method was implemented by incrementing $\lambda$ (the Lagrange multiplier) until a value was found that gave $|\mathbf{v}|=1$. The value of the Lagrange multipliers are: 1) 0.04432415474 and 2) 0.04432415661 . As can be seen it is only after the eighth decimal place that the two numbers differ. These numbers were confirmed by calculating the vector $\mathbf{v}$ and the resulting tilt and ellipticity angles and then comparing them to those obtained by using a simple search method to find the minimum of the 
copolar power function. These KPT copolar power minima, which can be difficult to find numerically, only exist because of the receive network modeled in KPT and do not exist in SNPT.

It was found that for all mean canting angles and variances of canting angle simulated, the tilt angle of the characteristic polarization state yielded the apparent mean canting angle, and that the accompanying ellipticity angle was zero for both SNPT and KPT. Several other particle types were also modeled, e.g., oblate ice and two layer (i.e., water coated ice), and again for various mean canting angles and variances of canting angles the cross-polar minimum power polarization states were always linear and indeed from the covariance matrices it was observed that in each case $\Im\left\{S_{\mathrm{HH}} S_{\mathrm{HV}}^{*}\right\}=$ $-\Im\left\{S_{\mathrm{HV}} S_{\mathrm{VV}}^{*}\right\}$.

\section{A. Mixed Precipitation}

Next, wet hail particles are added to the above described ensemble of rain particles in order to simulate a rain/hail mixture. Wet hail is modeled as oblate spheroids with an axis ratio of 0.75 and with the dielectric constant of water at $0^{\circ}$ centigrade. The raindrops are given a mean canting angle of $-20^{\circ}$ in the plane of polarization. The hailstones are assumed to follow an exponential size distribution of the form [27]

$$
N(D)=N_{0} e^{-\Lambda D}
$$

where $N_{0}=115 \Lambda^{3.63}, \Lambda$ is in units of $\mathrm{mm}^{-1}, N_{0}$ is in $\mathrm{mm}^{-1} \mathrm{~m}^{-3}$, and $D$ is in $\mathrm{mm}$. The distribution was truncated at $0.5 \mathrm{~cm}$ and $4.0 \mathrm{~cm}$. The mean tilt angle, $\bar{\theta}$, is $90^{\circ}$ (i.e., the major axis is vertical) with a standard deviation of $20^{\circ}$ and $\phi^{\prime}$ is distributed uniform in the interval $0-2 \pi$. Thus, the hail particles alone exhibit negative $Z_{\mathrm{DR}}$ (in the $\mathrm{H}-\mathrm{V}$ basis) and the $Z_{\mathrm{DR}}$ value is $-0.85 \mathrm{~dB}$. The reflectivity, $10 \log \left(\left\langle\left|S_{\mathrm{HH}}\right|^{2}\right\rangle\right)$, of the hail alone was made equal to the reflectivity of the rain component. Combining the hail with the rain yields the following covariance matrix:

$$
\mathbf{\Sigma}_{\mathbf{0}}=\left[\begin{array}{lll}
1.0 & 0.0907 e^{-j 171.4^{\circ}} & 0.8934 e^{j 0.39^{\circ}} \\
0.0907 e^{j 171.4^{\circ}} & 0.025 & 0.0583 e^{j 166.6^{\circ}} \\
0.8934 e^{-j 0.39^{\circ}} & 0.0583 e^{-j 166.6^{\circ}} & 0.8619
\end{array}\right]
$$

which is normalized by $\left\langle\left|S_{\mathrm{HH}}\right|^{2}\right\rangle$ with $10 \log \left(\left\langle\left|S_{\mathrm{HH}}\right|^{2}\right\rangle\right)=$ $60.3 \mathrm{dBZ}$. The optimum polarizations are are given in Table IV. For this covariance matrix $\Im\left\{S_{\mathrm{HH}} S_{\mathrm{HV}}^{*}\right\}=$ $-\Im\left\{S_{\mathrm{HV}} S_{\mathrm{VV}}^{*}\right\}$ and therefore the SNPT cross-polar minimum is linear; however, the cross-polar minimum for KPT is slightly elliptical with the angle of ellipticity being $-0.3^{\circ}$. Also, the copolar maximum polarization state no longer match the crosspolar minimum polarization state for either KPT or SNPT as was true for single distribution of particle type (i.e., only rain or hail or graupel etc.). The nonzero ellipticity angles for KPT are a result of modeling both the radar receive network as well as the rain/hail mixture in KPT. The nonmatching of the copolar maximum polarization state and cross-polar minimum polarization state is related to the mixing of different particle types with different orientation distributions thus creating a nonsymmetric orientation distribution of particles. The tilt

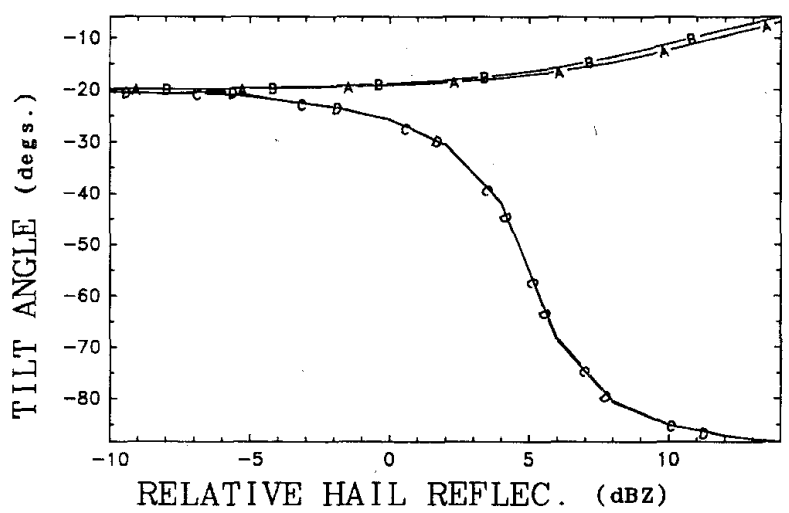

Fig. 5. The tilt angles for the characteristic polarization state and the copolar maximum polarization state as a function of the relative horizontal reflectivity of the hail, e.g., for $0 \mathrm{dBZ}$ the rain and hail ensembles have equal reflectivities. Curve A: KPT characteristic tilt. Curve B: SNPT characteristic tilt. Curve C: KPT copolar maximum tilt. Curve D: SNPT copolar maximum tilt.

angle of the cross-polar minimum polarization state for KPT and SNPT is $-18.8^{\circ}$ which is very close to the $-20.0^{\circ}$ mean canting angle given to the raindrops. This result shows that even with significant amounts of hail, the algorithm was able to resolve the apparent mean canting angle of the rain particles. If the number density of the hail particles is increased, thus increasing the hail reflectivity, the estimated mean canting angle will err further from $-20^{\circ}$ and likewise, if the hail density is decreased, better estimates of the mean canting angle of the raindrops can be expected. In other words, the estimated mean canting angle will be a reflectivity weighted average of the hail and rain mean canting angles. To illustrate this, the above ensembles of raindrops and hail particles are now mixed for various relative reflectivity values. Shown in Fig. 5 are the calculated mean tilt angles for the characteristic polarization basis and the copolar maximum polarization basis for both KPT and SNPT. For low relative hail reflectivities both the characteristic tilt angles and the copolar maximum tilt angles give good estimates of the mean canting angle of the raindrops. As the relative hail reflectivity increases, the characteristic tilt angle continues to give a reasonable estimate of the mean canting angle of the raindrops up to approximately $5 \mathrm{dBZ}$ relative hail reflectivity. However, the copolar tilt angle decreases rapidly toward $-90^{\circ}$ which is the mean canting angle of the hail particles. For this case the tilt angle of the characteristic polarization state is a better estimator of the mean canting angle of the raindrops. Fig. 6 shows the accompanying ellipticity angles for the KPT characteristic polarization state (Curve A) and the copolar maximum polarization state (Curve B). For all values of relative hail reflectivity the ellipticity angles are small being less than $1^{\circ}$. The ellipticity angles for SNPT are not shown since they are zero.

If the hail particles are distributed uniform random in both $\theta$ and $\phi^{\prime}$ (i.e., no preferential alignment) then much larger relative hail reflectivities will not affect the estimated mean canting angle of the raindrops using either the tilt angle from the characteristic polarization state or copolar maximum polarization state. When relative hail reflectivities as high as 


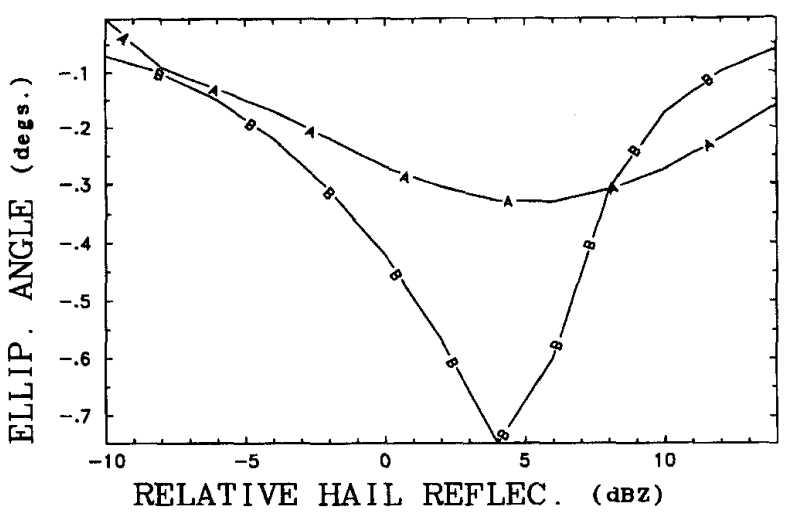

Fig. 6. The ellipticity angles for the characteristic polarization state and the copolar maximum polarization state as a function of the relative horizontal reflectivity of the hail, e.g., for $0 \mathrm{dBZ}$ the rain and hail ensembles have equal reflectivities. Curve $\mathbf{A}$ : ellipticity angle of KPT characteristic polarization state. Curve B: ellipticity angle of KPT copolar power maximum polarization state. The equivalent SNPT ellipticity angles are always zero.

TABLE IV

The Optimum Polarizations for the Rain/Hail Mixture. The Ensemble

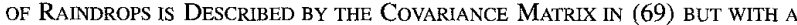

Mean Canting angle of $-20^{\circ}$ in the Plane of Polarization

\begin{tabular}{|c|c|c|c|c|c|}
\hline \multicolumn{6}{|c|}{ OPTIMUM POLARIZATIONS } \\
\hline & & \multicolumn{2}{|c|}{ KPT } & \multicolumn{2}{|c|}{ SNP'T $^{\prime}$} \\
\hline & & tilt & ellip. & tilt & ellip. \\
\hline \multirow{4}{*}{ COPOL } & $\max$. & $-25.6^{\circ}$ & $-0.24^{\circ}$ & $-25.6^{\circ}$ & $0^{\circ}$ \\
\hline & saddle & $57.2^{\circ}$ & $0.23^{\circ}$ & - & - \\
\hline & $\min .1$ & $64.2^{\circ}$ & $-42.8^{\circ}$ & $=$ & - \\
\hline & $\min .2$ & $58.3^{\circ}$ & $43.3^{\circ}$ & $57.2^{\circ}$ & $0^{\circ}$ \\
\hline \multirow{3}{*}{ XPOL } & $\max$. & $-8.58^{\circ}$ & $44.7^{\circ}$ & $0^{\circ}$ & $45^{\circ}$ \\
\hline & saddle & $26.2^{\circ}$ & $-0.11^{\circ}$ & $26.2^{\circ}$ & $0^{\circ}$ \\
\hline & $\min$. & $-18.8^{\circ}$ & $-0.30^{\circ}$ & $-18.8^{\circ}$ & $0^{\circ}$ \\
\hline
\end{tabular}

$35 \mathrm{dBZ}$ are used, the estimated canting angle is within a half of a degree of the actual raindrop mean canting angle of $-20^{\circ}$. To explain this, note that ensembles of particles which have a uniform orientation distribution have co-to-cross covariances that are are zero and therefore the co-to-cross covariances of the mixture are a function of the raindrops only. As another application, it is well known that small ice crystals can be oriented by in-cloud electric fields (giving a nonzero mean canting angle) while the larger snow particles are not and thus the larger particles are uniformly random distributed. Even if the large snow particles contribute 35 $\mathrm{dBZ}$ more relative reflectivity than the oriented ice crystals, it still should be possible to recover the mean canting angle of the ice crystals provided that an accurate measurement of the cross-polar signal can be made.

\section{B. Separation of Shape and Orientation}

In order to separate particle shape effects from the effects of orientation (i.e., spread of canting angle distribution) a technique suggested by [28] is used. They showed that the ratio of the maximum to minimum LDR value for linear polarizations is nearly independent of shape effects and depends primarily on orientation effects. The general linear depolarization ratio is defined here as $\operatorname{LDR}_{X}=10 \log \left[\left|S_{X Y} / S_{X X}\right|^{2}\right]$ where $X$ and $Y$ are any two orthogonal polarization states. Accordingly,

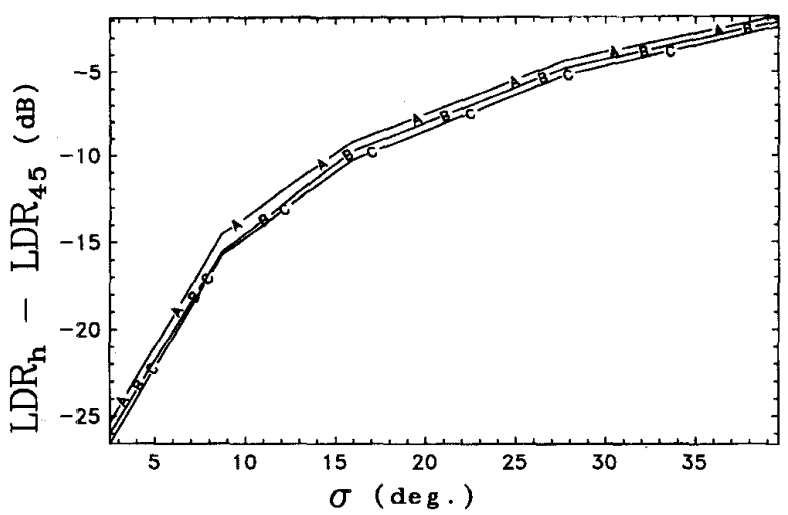

Fig. 7. $\mathrm{LDR}_{H}-\mathrm{LDR}_{45}$ as a function of the standard deviation of the canting angle distribution $\sigma$. Curves $\mathbf{A}, \mathbf{B}, \mathbf{C}$ correspond to drop size distributions truncated at $3,5.5$, and $7 \mathrm{~mm}$, respectively.

$\mathrm{LDR}_{H}$ and $\mathrm{LDR}_{45}$ represent the linear depolarization ratio in the $\mathrm{H}-\mathrm{V}$ and \pm 45 linear polarization bases. $\mathrm{LDR}_{45}$ can be obtained from $\Sigma$ in (20) by letting $\chi=1$.

Again, raindrops are modeled as before with exponential size distribution and a Fisher spatial distribution with a covariance matrix as given by (69). The upper limit of the drop size distribution is varied between $3-7 \mathrm{~mm}$ to simulate a variety of mean shapes which will vary the $Z_{\mathrm{DR}}$ of the ensemble. Shown in Fig. 7 is the difference $\mathrm{LDR}_{H}-\mathrm{LDR}_{45}$ as a function of the standard deviation of the canting angle distribution $\sigma$. Curves $\mathbf{A}, \mathbf{B}, \mathbf{C}$ correspond to the maximum drop size diameters of $3,5.5$, and $7 \mathrm{~mm}$, respectively. As can be seen the curves lie very close to one another even though $Z_{\mathrm{DR}}$ varies as much as $2.6 \mathrm{~dB}$ for a given value of $\sigma$ as is shown by Fig. 8 . If the particles have uniform orientation distribution them $\mathrm{LDR}_{H}-\mathrm{LDR}_{45}=0$ and $Z_{\mathrm{DR}}=0$. Since $\mathrm{LDR}_{H}-\mathrm{LDR}_{45}$ is nearly independent of $Z_{\mathrm{DR}}$, a reasonable estimate of the standard deviation of the canting angle can be made assuming that the particles follow a Fisher orientation distribution. If the particles have a mean canting angle other than horizontal, then the above polarimetric transformation can be applied so that the covariance matrix is expressed in its characteristic polarization basis. The mean axis ratio can be estimated from $Z_{\mathrm{DR}}$ in this basis.

\section{Symmetry and Optimum Polarizations}

It has been established [29] that for single scatterers possessing a line of symmetry (perpendicular to the line of sight) there exists a linear polarization state such that the cross-polar power is zero. Interestingly, this is equivalent to the condition (66) for coherent scattering and can be easily seen by examining the expression for the KPT cross-polar nulls for coherent reciprocal scattering, shown as (75), at the bottom of the next page. As can been seen the numerator is always real and if (66) is true then the denominator is also real. Thus $\chi^{x-n u l l}$ is real and the polarization state is linear. Condition (66) can also be derived from the expression for the cross-polar null polarization states for SNPT. But for an ensemble of particles, (66) only applies to the cross-polar power extrema for SNPT and is not a sufficient condition for cross-polar power extrema 


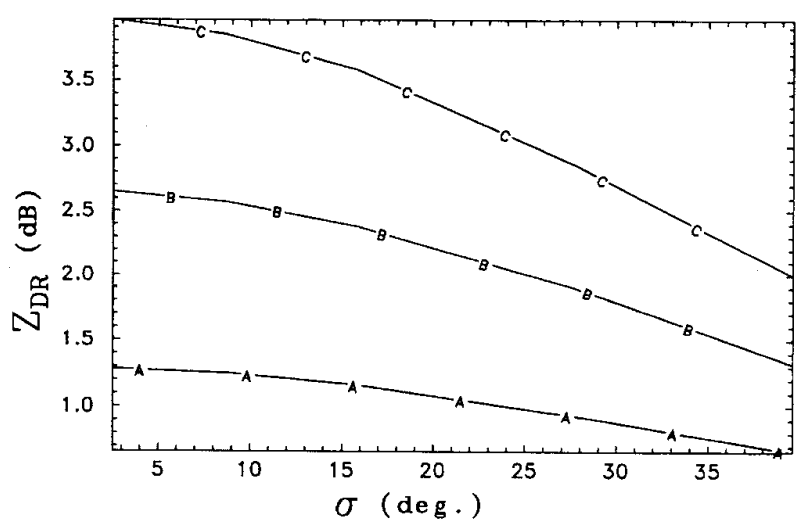

Fig. 8. $Z_{\mathrm{DR}}$ as a function of the standard deviation of the canting angle distribution $\sigma$. Curves $\mathbf{A}, \mathbf{B}, \mathbf{C}$ correspond to drop size distributions truncated at $3,5.5$, and $7 \mathrm{~mm}$, respectively.

for KPT. The above example of a mixture of hail and rain particles illustrated this fact. It is intuitively satisfying that the condition for linear cross-polar nulls for coherent scattering is identical to the condition for linear cross-polar extrema for incoherent scattering.

Our modeling studies show that if the particle orientation distribution is symmetric about some plane then the characteristic polarization basis (or cross-polar null polarization state) is linear and the tilt angle of the characteristic polarization state is the apparent mean canting angle of the ensemble of particles for both KPT and SNPT. If two particle ensembles with different orientation distributions are combined such that the composite ensemble is no longer symmetric, then the characteristic polarization state in KPT becomes elliptical although only slightly for the case examined in this paper. In contrast, the characteristic polarization state was linear for SNPT. We note that the characteristic polarization state in SNPT will always be linear for any ensemble that is composed of symmetric particles. We have observed that for any of the particles that are modeled by the $T$-matrix method, which must be rotationally symmetric, condition (66) is always satisfied. Since the covariance matrix is constructed by a weighted incoherent addition of the individual particles, condition (66) will also necessarily be satisfied for the ensemble regardless of the symmetry of the orientation distribution. A possible indicator of nonsymmetric orientation distribution is the degree of similarity of the SNPT copolar power maximum polarization state and characteristic polarization state.

\section{CONCLUSION}

In traditional radar polarimetry (KPT), both the scatterer and the transmit/receive network of the radar are modeled in the the change of basis formulation (2). Specifically, it is the modeling of the receive network of the radar that is responsible for the complex conjugation in the eigenvalue statement (14) and the transpose instead of inverse in (2). This modeling of the radar receive network affects optimum polarizations and polarization responses. For example if the copolar differential quantities $Z_{\mathrm{DR}}$ (differential reflectivity) or $\delta_{\mathrm{co}}$ (differential phase) are plotted as a function of incident tilt and ellipticity angles, large minima and maxima values result in the areas around the KPT copolar power minima (e.g., see Fig. 3). Specular null polarization theory (SNPT), which uses (9) for the definition of eigenpolarization, shows that these minima and maxima are a result of modeling the radar receive network in KPT.

SNPT does not include modeling of the radar receive network and as a consequence separates those characteristics that are due to the nature of the scatterer. This separation is accomplished by using a specular scatterer (for example, a spherical raindrop) as a reference scatterer. This means that that polarization state which results when a plane wave is specularly reflected is defined as the copolar reception polarization state. The resulting mathematics when expressed in the RAC takes a standard form: the eigenvalue statement is $\ddot{\mathbf{S}} \mathbf{x}=\lambda \mathbf{x}$ and the change of basis transformation is $\tilde{\ddot{\mathbf{S}}}=\mathbf{U}^{-1} \ddot{\mathbf{S}} \mathbf{U}$. This paper has extented this theory to incoherent scattering using a covariance matrix approach and the optimum polarizations were derived. The results for the reciprocal case show that there is only one value for the Lagrange multiplier that corresponds to a SNPT copolar elliptical optimum polarization and this optimum polarization can be found analytically. The remaining four SNPT optimum polarizations are always linear and are found numerically via the Lagrange multiplier method. This is in contrast to traditional radar polarimetry theory which yields six optimum copolar polarizations which may be either linear or elliptical. Our modeling studies for ensembles of spheroids indicate that there are only two linear SNPT copolar optimum polarizations, a maximum and a minimum, while there are four KPT copolar optimum polarizations, a maximum, two minima and a saddle point. The two additional KPT optimum polarizations, which are KPT copolar minima, are attributed to modeling the radar receive network. As in KPT there are three SNPT optimum cross-polar polarizations along with their orthogonal counterparts. However, in SNPT one cross-polar optimum polarization is always circular. The remaining two optimum polarizations will be linear if $\Im\left\{\left\langle S_{\mathrm{HH}} S_{\mathrm{HV}}^{*}\right\rangle\right\}=-\Im\left\{\left\langle S_{\mathrm{HV}} S_{\mathrm{VV}}^{*}\right\rangle\right\}$, (66), which is not true for KPT. It is intuitively satisfying that the condition for crosspolar linear optimum polarization for incoherent scattering is the same condition as for coherent scattering in SNPT. The zero entries in the Hermitian quadritic forms for copolar and cross-polar power for SNPT reduce the complexity of calculating optimum polarizations for SNPT in comparison to KPT.

Ensembles of particles were modeled using the transition $(T-)$ matrix as was done by [21]. Their method was extended

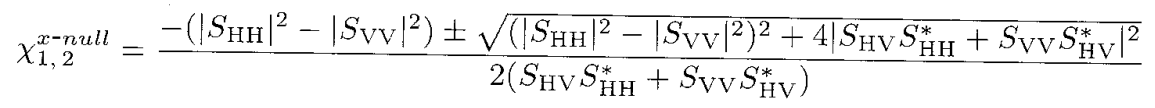


to allow for arbitrary mean canting angles which was accomplished by using a Fisher distribution [23] to model the particle orientation distribution. When a single class of particles, such as raindrops, was modeled with a Fisher distribution, the tilt angle from the cross-polar power minimum polarization (the characteristic polarization state or basis) was the mean canting angle if the ensemble mean canting angle was in the plane of polarization; otherwise the tilt angle from the crosspolar power minimum polarization was the apparent mean canting angle which is that angle that results when the mean canting angle of the ensemble is projected on to the plane of polarization. If two particle ensembles, such as raindrops and hailstones, have different orientation distributions, then the tilt angle from the cross-polar power minimum polarization will be, in general, a weighted average of the mean canting angle from each particle ensemble. If, however, one particle ensemble is distributed uniformly random in space while the other possesses a mean canting angle, then it is possible to detect the mean canting angle even if the reflectivity of the randomly oriented particles exceeds the reflectivity of the oriented particles by $30-35 \mathrm{dBZ}$. For the mixture of hail and rain example given in this paper, the tilt angle from the cross-polar power minimum polarization was a better estimator of the mean canting angle of the raindrops than the tilt angle from the copolar maximum polarization. Since for ensembles of spheroids (66) is always true, the SNPT crosspolar minimum polarization state will always be linear for this class of scatterers. This is not necessarily true for KPT as the hail/rain ensemble illustrated. For all of the various mixtures of spheroids we have modeled, the SNPT copolar optimum polarizations were always linear, i.e., constraint (45) was never satisfied when the Lagrange multiplier $\lambda=$ $W_{33}$. Therefore, we believe that for ensembles composed of rotationally symmetric particles, the SNPT copolar optimum polarization are always linear. Our modeling results also suggest that for ensemble of spheriods there exist only two SNPT copolar optimum polarizations both of which are linear while there are four KPT copolar optimum polarizations of which two are typically elliptical. We believe that the two additional KPT copolar optimum polarizations and the ellipticity angles of the optimum polarizations exist only due to the modeling of the radar receive network in the KPT equations.

Our modeling results also showed that it is possible to separate orientation effects from shape effects as was suggested by [28]. This is done by calculating LDR (linear depolarization ratio) in the characteristic polarization basis and also in the linear basis $45^{\circ}$ in tilt angle from the characteristic polarization basis. The difference of these two LDR's is a good estimator of the spread of the canting angle distribution.

For meteorological applications, we then suggest the following steps.

1) Accurately measure the backscatter covariance matrix in the $\mathrm{H}-\mathrm{V}$ basis.

2) Calculate the characteristic polarization state from which the mean canting angle is estimated.

3) Transform the covariance matrix to the characteristic polarization basis.
4) Calculate the relevant radar parameters e.g., $Z_{\mathrm{DR}}, \delta_{\mathrm{co}}, \rho_{\mathrm{co}}$ in this basis. These parameters can be used to infer the mean shape, Mie scattering effects and particle mixtures.

5) Transform the covariance matrix to the linear basis which is $45^{\circ}$ from the characteristic polarization basis and calculate LDR in that basis. Difference this LDR with the LDR in the characteristic polarization basis to obtain an estimate of the canting angle spread.

Finally, we note that SNPT is equivalent to treating the complex backscattering amplitudes as if they were complex forward scattering amplitudes and then analyzing the backscatter problem as a forward scattering problem with the additional restriction that $S_{\mathrm{HV}}=S_{\mathrm{VH}}$. Therefore, SNPT presented here for the case where $S_{\mathrm{HV}} \neq S_{\mathrm{VH}}$ may also be applied to general forward or bistatic scattering problems.

\section{APPENDIX A}

\section{DERIVATION OF POLARIZATION TRANSFER FUNCTION}

To obtain the equation that relates incident and scattered polarization ratios in the $L R H$, we begin with the optic scattering matrix equation in the $\mathrm{H}-\mathrm{V}$ (horizontal and vertical) basis

$$
\left[\begin{array}{c}
E_{H^{\prime}}^{s} \\
E_{V^{\prime}}^{s}
\end{array}\right]=\left[\begin{array}{ll}
\dot{S}_{\mathrm{HH}} & \dot{S}_{\mathrm{HV}} \\
\dot{S}_{\mathrm{VH}} & \dot{S}_{\mathrm{VV}}
\end{array}\right]\left[\begin{array}{c}
E_{H}^{i} \\
E_{V}^{i}
\end{array}\right]
$$

where $\dot{S}$ are the members of the optic (Jones) scattering matrix and are related to the radar (Sinclair) scattering matrix by

$$
\dot{\mathbf{S}}=\left[\begin{array}{rr}
-1 & 0 \\
0 & 1
\end{array}\right] \mathbf{S}
$$

which is valid for relating the $\mathrm{H}-\mathrm{V}$ components of the two matrices only. Using this relationship, the matrix $\dot{\mathbf{S}}$ will be written as

$$
\dot{\mathbf{S}}=\left[\begin{array}{rr}
-S_{\mathrm{HH}} & -S_{\mathrm{HV}} \\
S_{\mathrm{VH}} & S_{\mathrm{VV}}
\end{array}\right]
$$

Substituting (A3) into (A1) gives

$$
\left[\begin{array}{l}
E_{H^{\prime}}^{s} \\
E_{V^{\prime}}^{s}
\end{array}\right]=\left[\begin{array}{rr}
-S_{\mathrm{HH}} & -S_{\mathrm{HV}} \\
S_{\mathrm{VH}} & S_{\mathrm{VV}}
\end{array}\right]\left[\begin{array}{l}
E_{H}^{i} \\
E_{V}^{i}
\end{array}\right] .
$$

Using this matrix equation it is simple to solve for the bilinear polarization transfer function (7).

\section{APPENDIX B}

\section{COMPARISON OF VOLTAGE EQUATIONS}

We compare the three voltage equations and show how the three different change of polarization basis formulas result.

$$
\begin{array}{ll}
\mathcal{V}=\mathbf{f}^{\dagger} \mathbf{E}_{\mathrm{LRH}}^{s} & \text { optic polarimetry } \\
\mathcal{V}=\mathbf{h}_{r}^{T} \mathbf{E}_{\mathrm{RAC}}^{s} & \text { radar polarimetry } \\
\mathcal{V}=\mathbf{g}^{\dagger} \mathbf{E}_{\mathrm{RAC}}^{s} & \text { SNPT }
\end{array}
$$

where $\dagger$ denotes Hermitian adjoint (transpose conjugate). The difference between the three equations can be understood by examining the definition of the reception polarization vectors $\mathbf{f}, \mathbf{h}$, and $\mathbf{g}$. In optic polarimetry $\mathbf{f}$ denotes a unit polarization 
reception vector defined in a $\mathrm{LRH}$. Since the incoming wave is also defined in the same coordinate system, i.e., LRH, a standard complex inner product rule is employed to find the received voltage. In radar polarimetry $h$ actually denotes the transmit polarization state of the receiving radar in the RAC. One must realize that if a radar transmits $\chi$ polarization than it receives $\chi^{*}$ when the RAC is used. This is why $\mathrm{h}^{T}$ is used instead of $\mathbf{h}^{\dagger}$ in the radar voltage equation and this is how modeling the radar is included in the radar voltage equation. In SNPT g denotes a unit polarization reception vector defined in the RAC. Since the incoming wave is also defined in the RAC, again a standard complex inner product rule is used to calculate the voltage.

The above voltage equations can be expanded to include the scattering matrices:

$$
\begin{array}{ll}
\mathcal{V}=\mathbf{f}^{\dagger} \dot{\mathbf{S}} \mathbf{h} & \text { optic polarimetry } \\
\mathcal{V}=\mathbf{h}_{r}^{T} \mathbf{S h} & \text { radar polarimetry } \\
\mathcal{V}=\mathrm{g}^{\dagger} \ddot{\mathbf{S}} \mathbf{h} & \text { SNPT }
\end{array}
$$

where $\mathbf{h}$ denotes the field incident on the scatterer. From (B4)-(B6) the change of basis formulas can be derived. Note that even though different coordinate conventions are used, all polarization vectors transform acording to the same rule: $\mathbf{f}=\mathbf{U f}^{\prime}, \mathbf{h}_{r}=\mathbf{U h}_{r}^{\prime}, \mathbf{g}=\mathbf{U g}^{\prime}$, and $\mathbf{h}=\mathbf{U h}^{\prime}$ where denotes a new polarization basis, [see (3) for a definition of U.] Substituting these into (B4), (B5), and (B6) gives the change of basis formulas $\dot{\mathbf{S}}^{\prime}=\mathrm{U}^{-1} \dot{\mathbf{S}} \mathbf{U}, \mathbf{S}^{\prime}=\mathrm{U}^{\mathbf{T}} \mathbf{S U}$, and $\ddot{\mathbf{S}}^{\prime}=\mathbf{U}^{-1} \ddot{\mathbf{S}} \mathbf{U}$, respectively.

\section{APPENDIX C}

\section{The Fisher Distribution}

The functional form is

$$
\begin{aligned}
g_{\kappa}\left(\theta, \phi^{\prime}\right)= & c \exp [\kappa\{\cos \bar{\theta} \cos \theta \\
& \left.\left.+\sin \bar{\theta} \sin \theta \cos \left(\phi^{\prime}-\bar{\phi}^{\prime}\right)\right\}\right] \sin \theta \\
& 0<\theta<\pi, 0<\phi^{\prime}<2 \pi, \text { and } \kappa>0
\end{aligned}
$$

where $\theta, \phi^{\prime}$ are the latitude and longitude angles of a spherical coordinate system, $\bar{\theta}, \bar{\phi}^{\prime}$ specify the mean canting direction, $\kappa$ controls the spread of the distribution and

$$
c=\frac{\kappa}{4 \pi \sinh \kappa} .
$$

In order to show the relationship between the parameter $\kappa$ and the spread of the Fisher distribution, $\bar{\phi}^{\prime}$ and $\bar{\theta}$ are taken as zero and the distribution function is integrated over $\phi^{\prime}$ and $\theta$ and equated to one standard deviation, $68.27 \%$

$$
\begin{aligned}
G(\theta) & =c \int_{0}^{2 \pi} \int_{0}^{\theta} e^{\kappa \cos \vartheta} \sin \vartheta d \vartheta d \phi^{\prime} \\
& =0.6827 .
\end{aligned}
$$

Solving for $\theta$ gives

$$
\theta=\arccos \left(\frac{\log \left\{e^{\kappa}\left[1-0.6827\left(1-e^{-2 \kappa}\right)\right]\right\}}{\kappa}\right)
$$

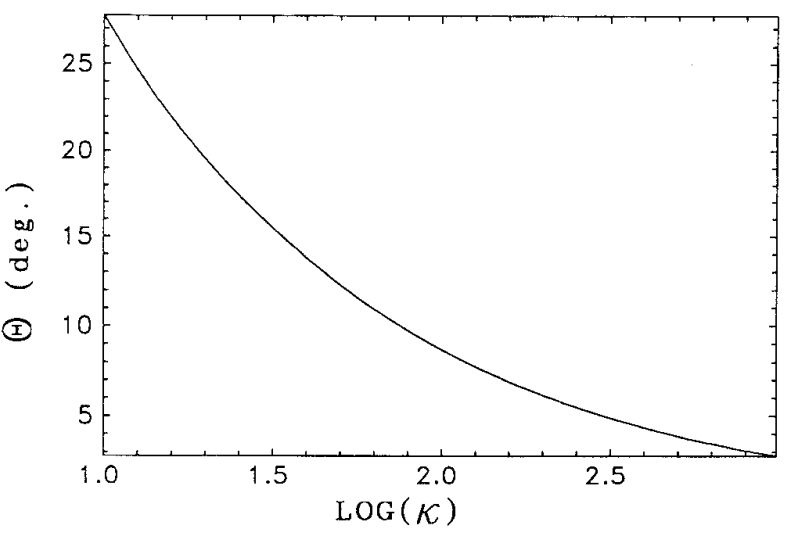

Fig. 9. Values of $\kappa$ for the fisher distribution versus solid angle $\theta$ with in which lie $68.27 \%$ of the canting angles of the particles.

where $\theta$ is the solid angle within which $68.27 \%$ of the particles lie. If $\kappa \geq 3$ (values of $\theta$ less than $\approx 52^{\circ}$ ), (C4) simplifies and $\kappa$ can be directly approximated by

$$
\kappa \approx \frac{1.1479}{1-\cos \theta}
$$

In other words, if one wishes to restrict $68.27 \%$ of the canting angles of the particles to within a solid angle of $52^{\circ}$ or less, then $\kappa$ may be found directly with (C5); otherwise (C4) should be used. Shown in Fig. 9 is a plot of (C4) with $\theta$ on the vertical axis and the base ten logarithm of $\kappa$ on the horizontal axis.

\section{REFERENCES}

[1] E. M. Kennaugh, "Effects of type of polarization on echo charac teristics," Antennas Lab., Ohio State Univ., Columbus, Tech. Rep. 381-1-39424, pp. 1949-1954

[2] F. T. Ulaby and J. J. van Zyl, "Scattering matrix representation for simple targets," in Radar Polarimetry for Geoscience Applications, F. T. Ulaby and C. Elachi, Eds. Norwood, MA: Artech House, 1990, ch. 1 and 2, pp. $1-52$.

[3] A. B. Kostinski and W.-M. Boerner, "On foundations of radar poIarimetry," IEEE Trans. Antennas Propagat., vol. AP-34, no. 12, pp. 1395-1404, Dec. 1986.

[4] A. P. Agrawal and W.-M. Boerner, "Redevelopment of Kennaugh's target characteristic polarization state theory using the polarization transformation ratio formalism for the coherent case," IEEE Trans. Geosci. Remote Sensing, vol. 27, pp. 2-14, Jan. 1989.

[5] J. C. Hubbert, "A comparision of radar, optic and specular null polarization theories," IEEE Trans. Geosci. Remote Sensing, vol. 32, no. 3, pp. 658-671, May 1994.

[6] M. Born and E. Wolf, Principles of Optics, 5th ed. New York: Pergamon, 1975

[7] R. M. A. Azzam and N. M. Bashara, Ellipsometry and Polarized Light. Amsterdam: North Holland, 1989.

[8] H. Mieras, "Comments on foundations of radar polarimetry," IEEE Trans. Antennas Propagat., vol. AP-34, no. 12, pp. 1395-1404, Dec. 1986.

[9] A. B. Kostinski and W.-M. Boerner, "Authors' reply," IEEE Trans. Antennas Propagat., vol. AP-34, no. 12, pp. 1470-1471, Dec. 1986.

[10] K. Tragl, "Polarimetric radar backscattering from reciprocal random targets," IEEE Trans. Geosci. Remote Sensing, vol. 8, no. 5, pp. 856-864, Sept. 1990.

[11] K. Tragl, K. E. Lüneburg, A. Schroth, and V. Ziegler, "A polarimetric covariance matrix concept for random radar targets," in Preprints, Int Conf. Antennas and Propagation, York, U.K., 1991, vol. IEE Publ. 333 of 7 th series, pp. 396-399.

[12] E. Lüneburg, V. Ziegler, A. Schroth, and K. Tragl, "Polarimetric covariance matrix analysis for random radar targets," in AGARD_Electromag. Wave Prop. Panel Symp. Target and Clutter Scat. and their Effects on Military Radar Performance, Ottawa, Canada, 1991, vol. CP-501, pp. 27-1-27-12 
[13] P. C. Waterman, "Scattering by dielectric particles," Alta Frequeza (Speciale), pp. 348-352, 1969 .

[14] S. Y. Matrosov and R. A. Kropfli, "Cirrus cloud studies with elliptically polarized Ka-band radar signals: A suggested approach," J. Atmos. Ocean. Tech., vol. 10, pp. 684-692, Oct. 1993.

[15] R. Kwok, E. J. M. Rignot, J. Way, and A. Freeman, "Polarimetric signatures of frozen and thawed forests of varying environmental state," IEEE Trans. Geosci. Remote Sensing, vol. 2, no. 5, pp. 371-381, Mar 1994.

[16] J. K. Lee and S. Mudaliar, "Optimum polarizations in the bistatic scattering from layered random media," IEEE Trans. Geosci. Remote Sensing, vol. 32, no. 1, pp. 169-175, Jan. 1994.

[17] J. M. Kwiatkowski, A. B. Kostinski, and A. R. Jameson, "The use of optimal polarizations for studying the microphysics of precipitation: Nonattenuatiing wavelengths," J. Atmos. Oceanic Tech., vol. 12, no. 1, pp. 96-114, Feb. 1995.

[18] M. R. Hestenes, Optimization Theory. Huntington, New York: Krieger, 1981.

[19] J. J. van Zyl, C. H. Papas, and C. Elachi, "On the optimum polarizations of incoherently reflected waves," IEEE Trans. Antennas Propagat., vol. AP-35, no. 7, pp. 818-825, 1987 .

[20] A. B. Kostinski, B. D. James, and W.-M. Boerner, "Optimal reception of partially polarized waves," J. Opt. Soc. Amer. A, vol. 5, no. 1, pp. 58-64, Jan. 1988.

[21] J. Vivekanandan, R. Raghavan, and V. N. Bringi, "Polarimetric radar modeling of mixtures of precipitation particles," IEEE Trans. Geosci. Remote Sensing, vol. 31, no. 5, pp. 1017-1030, Sept. 1993.

[22] R. A. Fisher, "Dispersion on a sphere," in Proc. Roy. Soc. Lond., 1953, vol. A217, pp. 295-305.

[23] K. V. Mardia, Statistics of Directional Data. New York and London: Academic, 1972

[24] N. Balakrishnan and D. S. Zrnić, "Use of polarization to characterize precipitation and discriminate large hail," J. Atmos. Sci., vol, 47, pp $1525-1540,1990$.

[25] D. S. Zrnić, V. N. Bringi, N. Balakrishnan, K. Aydin, V. Chandrasekar, and J. Hubbert, "Polarimetric measurements in a severe hailstorm," Monthly Weather Rev., vol. 121, no. 8, pp. 2223-2238, Aug. 1993.

[26] A. B. Kostinski, "Fluctuations of differential phase and radar measurements of precipitation," J. Appl. Meteor., vol. 33, pp 1176-1181, Oct 1994

[27] L. Cheng and M. English, "A relationship between hailstone concentration and size," J. Atmos. Sci., vol. 40, pp. 204-213, 1983.
[28] A. Hendry, Y. M. M. Antar, and G. C. McCormick, "On the relationship between the degree of preferred orientation in precipitation and dual polarization radar echo characteristics," Radio Sci., vol. 22, pp. 37-50, 1987. (Also, see correction, p. 687.) Radio Sci., vol. 22, no. 4, p. 687.

[29] J. R. Huynen, "Phenomenological theory of radar targets," Ph.D. dissertation, Tech. Univ. Delft, Delft, The Netherlands, 1970.

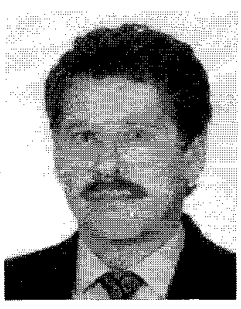

John C. Hubbert received the B.A. degree (1974) in mathematics from the University of Vermont Burlington, and the M.S. (1985) and Ph.D. (1992) degrees in electrical engineering from Colorado State University, Fort Collins.

From 1985 to 1986, he was with Hewlett-Packard in Boeblingen, West Germany, developing low noise analog amplifiers for biomedical applications. He is presently with Colorado State University in the CSU-CHILL remote sensing laboratory. His research interests are theoretical polarimetry, scattering theory, digital filtering, precipitation modeling, and radar polarimetry applied to cloud microphysics.

V. N. Bringi obtained the B.Tech. (Hons.) degree in electrical engineering from the Indian Institute of Technology, Bombay, India, in 1971, and the $\mathrm{Ph} . \mathrm{D}$. degree in electrical engineering from Ohio State University, Columbus, in 1976 .

He joined Colorado State University in 1981 as an Associate Professor and was promoted to the rank of Full Professor in 1987. His research interests are in application of radar polarimetry to meteorology. He has served on the American Meteorological Society's Committee on Radar Meteorology. He is currently an Associate Editor of the Journal of Atmospheric and Oceanic Technology. He is Co-Principal Investigator for the CSU-CHILL national radar facility operated by Colorado State University under a five-year cooperative agreement with the National Science Foundation. 